\title{
QUASI-LOCAL ALGEBRAS AND ASYMPTOTIC EXPANDERS
}

\author{
KANG LI, PIOTR NOWAK, JÁN ŠPAKULA AND JIAWEN ZHANG
}

\begin{abstract}
In this paper, we study the relation between the uniform Roe algebra and the uniform quasi-local algebra associated to a metric space of bounded geometry. In the process, we introduce a weakening of the notion of expanders, called asymptotic expanders. We show that being a sequence of asymptotic expanders is a coarse property under certain connectedness condition, and it implies non-uniformly local amenability. Moreover, we also analyse some $C^{*}$-algebraic properties of uniform quasi-local algebras. In particular, we show that a uniform quasi-local algebra is nuclear if and only if the underlying metric space has Property A.
\end{abstract}

Mathematics Subject Classification (2010): 46H35, 46L05, 20F65, 05 C99.

\section{INTRODUCTION}

(Uniform) Roe algebras are $C^{*}$-algebras associated to discrete metric spaces, which reflect and encode the coarse (or large-scale) geometry of the underlying metric spaces. They have been well-studied and have fruitful applications, among which the most important ones would be the (uniform) coarse Baum-Connes conjecture, the Novikov conjecture, the zero-in-the-spectrum conjecture and the conjecture of positive scalar curvature on manifolds (e.g. [30, 32, 41, 42, 43, 44, 45]).

Recently, there has been substantial research about the interplay between coarsegeometric properties of a metric space $X$ with bounded geometry and analytic properties of its uniform Roe algebra $C_{u}^{*}(X)$ (e.g. [1, 2, 8, 17, 18, 30, 36, 40]). A prototypical result in this direction comes from [13, 21, 30]: a metric space $X$ has Property $\mathrm{A}$ if and only if $C_{u}^{*}(X)$ is a nuclear $C^{*}$-algebra.

A fundamental question is to determine whether a given operator belongs to the uniform Roe algebra. To overcome this issue, Roe suggested the notion of quasilocality in [23, 24] and observed that operators in uniform Roe algebras are always quasi-local. The converse is open, although it has been proven under additional assumptions on the underlying spaces [10, 33, 34]. This piece revolves around comparing the uniform Roe algebra $C_{u}^{*}(X)$ of a bounded geometry metric space $X$ with the $C^{*}$-algebra $C_{u q}^{*}(X)$ of all quasi-local operators in $\mathfrak{B}\left(\ell^{2}(X)\right)$ (see Definition 2.1 and Definition 2.2 for the definition of the uniform quasi-local algebra $\left.C_{u q}^{*}(X)\right)$. We always have $C_{u}^{*}(X) \subseteq C_{u q}^{*}(X)$, and if the space $X$ has Property $\mathrm{A}$, then we have the equality $C_{u}^{*}(X)=C_{u q}^{*}(X)[34$, Theorem 3.3(2)].

Date: April 2, 2020.

Key words and phrases. Expanders, Nuclearity, Property A, Quasi-local algebras.

KL and PN were supported by the European Research Council (ERC) under the European Union's Horizon 2020 research and innovation programme (grant agreement no. 677120-INDEX). JŠ was partially supported by Marie Curie FP7-PEOPLE-2013-CIG Coarse Analysis (631945). JZ was supported by the Sino-British Trust Fellowship by Royal Society, International Exchanges 2017 Cost Share (China) grant EC $\backslash$ NSFC $\backslash 170341$, and NSFC11871342. 
The motivation for this paper is to look for obstructions to this equality. More precisely, we attempt to tell the difference between $C_{u}^{*}(X)$ and $C_{u q}^{*}(X)$ via the averaging projection $P_{X} \in \mathfrak{B}\left(\ell^{2}(X)\right)$ over the coarse disjoint union $X=\bigsqcup_{n \in \mathbb{N}} X_{n}$ of a sequence of finite metric spaces $\left\{X_{n}\right\}_{n \in \mathbb{N}}$ (see Definition 3.4). It is well known that if $X$ is an expander, then $P_{X} \in C_{u}^{*}(X)$ (see also the discussion before Definition 3.4). On the other hand, $P_{X} \notin C_{u}^{*}(X)$ if $X$ can be coarsely embedded into some Hilbert space according to Finn-Sell's work [11, Proposition 35]. Hence, it is crucial to know when the averaging projection $P_{X}$ belongs to $C_{u q}^{*}(X)$. It turns out that the quasi-locality of $P_{X}$ is equivalent to $\left\{X_{n}\right\}_{n \in \mathbb{N}}$ being a sequence of asymptotic expanders, which is a slight weakening of being an expander sequence.

Definition A (Definition 3.12). A sequence of finite metric spaces $\left\{X_{n}\right\}_{n \in \mathbb{N}}$ with $\left|X_{n}\right| \rightarrow \infty$ (as $n \rightarrow \infty$ ) is said to be a sequence of asymptotic expanders if for any $\alpha>0$, there exist $c \in(0,1)$ and $R>0$ such that for any $n \in \mathbb{N}$ and $A \subseteq X_{n}$ with $\alpha\left|X_{n}\right| \leq|A| \leq\left|X_{n}\right| / 2$, we have $\left|\partial_{R} A\right|>c|A|$, where $\partial_{R} A:=\left\{x \in X_{n} \backslash A: d(x, A) \leq\right.$ $R\}$.

We prove the following statement:

Theorem B (Theorem 3.11). Let $\left\{X_{n}\right\}_{n \in \mathbb{N}}$ be a sequence of finite metric spaces with $\left|X_{n}\right| \rightarrow \infty$ as $n \rightarrow \infty$. Let $X=\bigsqcup_{n \in \mathbb{N}} X_{n}$ be a coarse disjoint union, and let $P_{X}$ the averaging projection of the sequence $\left\{X_{n}\right\}_{n \in \mathbb{N}}$. Then $P_{X}$ is quasi-local if and only if $\left\{X_{n}\right\}_{n \in \mathbb{N}}$ is a sequence of asymptotic expanders.

Remark 1.1. While we assume bounded geometry throughout the paper, the notions of quasi-locality and asymptotic expanders themselves are meaningful also when $X$ does not have bounded geometry. Our proof of the above theorem does not require bounded geometry, and thus in Subsection 3.2 we only assume that the metric spaces are discrete.

Therefore, the existence of a sequence of asymptotic expanders whose coarse disjoint union $X$ can be coarsely embedded into some Hilbert space would imply that the associated averaging projection $P_{X}$ is quasi-local but does not belong to the uniform Roe algebra of $X$. In other words, $C_{u}^{*}(X) \varsubsetneqq C_{u q}^{*}(X)$ (see Proposition [7.4). However, we did not yet succeed in finding such an example of $X$ (see Question 7.3).

Asymptotic expanders themselves might be of independent interest to experts in graph theory (see Theorem 3.11 for different formulations similar to the Cheeger constant of expanders). We show that asymptotic expanders are strictly more general than expanders (see Corollary 3.13). Moreover, we study coarse properties of asymptotic expanders, showing that being a sequence of asymptotic expander graphs is invariant under coarse equivalences, and is incompatible with uniformly local amenability:

Theorem C (Corollary 3.16). Let $\left\{X_{n}\right\}_{n \in \mathbb{N}}$ and $\left\{Y_{n}\right\}_{n \in \mathbb{N}}$ be sequences of finite connected graphs with bounded valency, such that $\left|X_{n}\right|,\left|Y_{n}\right| \rightarrow \infty$ as $n \rightarrow \infty$. If coarse disjoint unions $X=\bigsqcup_{n \in \mathbb{N}} X_{n}$ and $Y=\bigsqcup_{n \in \mathbb{N}} Y_{n}$ are coarsely equivalent, and $\left\{X_{n}\right\}_{n \in \mathbb{N}}$ is a sequence of asymptotic expanders, then so is $\left\{Y_{n}\right\}_{n \in \mathbb{N}}$.

Theorem D (Theorem 4.4). Let $X$ be a metric space with bounded geometry, which is a coarse disjoint union of a sequence of asymptotic expanders. Then $X$ is not uniformly locally amenable. In particular, $X$ does not have Property $A$. 
Finally, we study $C^{*}$-algebraic properties of the uniform quasi-local algebra $C_{u q}^{*}(X)$ of a metric space with bounded geometry. As alluded to above, we already know that $X$ having Property $\mathrm{A}$ is equivalent to the nuclearity of its uniform Roe algebra $C_{u}^{*}(X)$, and when $X$ has Property A we have $C_{u}^{*}(X)=C_{u q}^{*}(X)$. Concerning uniform quasi-local algebras, we are able to prove the following theorem:

Theorem E (Theorem 5.5 and Proposition 6.1). Let X be a metric space of bounded geometry. Then

- $X$ has Property $A$ if and only if the uniform quasi-local algebra $C_{u q}^{*}(X)$ is nuclear;

- $C_{u}^{*}(X)=C_{u q}^{*}(X)$ if and only if $\ell^{\infty}(X) \subseteq C_{u q}^{*}(X)$ is a Cartan subalgebra.

In particular, Theorem E shows that we can not use nuclearity to distinguish $C_{u q}^{*}(X)$ from $C_{u}^{*}(X)$. Moreover, we know that $\ell^{\infty}(X) \subseteq C_{u}^{*}(X)$ is always a Cartan subalgebra, and structural and uniqueness questions for Cartan subalgebras in uniform Roe algebras were intensively studied in [37.

The paper is organised as follows. In Section 2, we recall some basic notions in coarse geometry which are used throughout the paper. In Section 3, we introduce the notion of asymptotic expanders, and prove Theorem B and Theorem C. We provide a proof of Theorem $\mathrm{D}$ in Section 4 . Moreover, Section 5 and Section 6 are devoted to Theorem E. Finally, we raise several open questions in Section 7.

Acknowledgments. We would like to thank Rufus Willett for sharing a draft on the quasi-locality of averaging projections. We would also like to thank Hiroki Sako for bringing Example 3.7 to our attention, and Baojie Jiang for several illuminating discussions. Finally, we would like to thank the anonymous referee for pointing a mistake in the statement of Theorem 3.15 of an early version, and many useful suggestions to make the paper more readable.

\section{Preliminaries}

Let $(X, d)$ be a metric space, $x \in X$ and $R>0$. Denote $B(x, R)$ the closed ball in $X$ with centre $x$ and radius $R$. For any $A \subseteq X$, denote by $|A|$ the cardinality of $A$, $\mathcal{N}_{R}(A)=\{x \in X: d(x, A) \leq R\}$ the $R$-neighbourhood of $A$, and $\partial_{R} A=\{x \in X \backslash A:$ $d(x, A) \leq R\}$ the (outer) $R$-boundary of $A$. Recall that a metric space $(X, d)$ has bounded geometry if $\sup _{x \in X}|B(x, R)|$ is finite for each $R>0$; 1 we shall occasionally use the notation $N_{X}(R)=\sup _{x \in X}|B(x, R)|$. We say that $X$ is D-connected (for some $D \geq 0$ ), if for all $x, y \in X$ there exists a sequence $x=x_{0}, x_{1}, \ldots, x_{n}=y$ of points in $X$, such that $d\left(x_{i-1}, x_{i}\right) \leq D$ for all $i \in\{1, \ldots, n\}$.

For $A \subseteq X$, we use $\chi_{A}$ for the characteristic function of $A$ and the symbol $\delta_{x}$ for $\chi_{\{x\}}$ for $x \in X$. By a slight abuse of notation, we shall use the functions $\chi_{A}$ both as vectors in $\ell^{2}(X)$ (when $A$ is finite), and as multiplication operators on $\ell^{2}(X)$ in the sense below defined.

Throughout this paper, unless stated otherwise, $(X, d)$ denotes a metric space with bounded geometry. The notable exception is Subsection 3.2. where we only require $X$ to be discrete.

\footnotetext{
${ }^{1}$ It is worth noticing that a metric space with bounded geometry must be discrete.
} 
2.1. Uniform Roe algebras. An operator $T \in \mathfrak{B}\left(\ell^{2}(X)\right)$ can be viewed as an $X$ by- $X$ matrix $\left[T_{x, y}\right]_{x, y \in X}$ with $T_{x, y}=\left\langle T \delta_{y}, \delta_{x}\right\rangle \in \mathbb{C}$. We say that $T \in \mathfrak{B}\left(\ell^{2}(X)\right)$ has finite propagation if there exists some constant $R>0$ such that $T_{x, y}=0$ if $d(x, y)>$ $R$. The smallest number $R$ satisfying this condition is called the propagation of $T$.

There are two elementary classes of operators with finite propagation: multiplication operators and partial translations: let $f \in \ell^{\infty}(X)$, the pointwise multiplication provides an operator in $\mathfrak{B}\left(\ell^{2}(X)\right)$ with zero propagation, called the multiplication operator of $f$ and still denoted by $f$ for simplicity. For the latter, let $D, R \subseteq X$ and $\theta: D \rightarrow R$ be a bijection. Define a matrix $V^{\theta}$ by

$$
V_{x, y}^{\theta}= \begin{cases}1, & x \in D \text { and } y=\theta(x), \\ 0, & \text { otherwise. }\end{cases}
$$

If $\sup _{x \in D} d(x, \theta(x))$ is finite, then $V^{\theta}$ is a partial isometry in $\mathfrak{B}\left(\ell^{2}(X)\right)$ with finite propagation and $V^{\theta}$ is called a partial translation (operator). It is direct to check that the set of all finite propagation operators in $\mathfrak{B}\left(\ell^{2}(X)\right)$ forms a *-algebra, called the algebraic uniform Roe algebra $\mathbb{C}_{u}[X]$ of $X$. It is well known that $\mathbb{C}_{u}[X]$ is generated by multiplication operators and partial translations as a $*$-algebra for every metric space $X$ with bounded geometry (see [25, Lemma 4.27]). The uniform Roe algebra $C_{u}^{*}(X)$ of $X$ is the operator-norm closure of $\mathbb{C}_{u}[X]$ inside $\mathfrak{B}\left(\ell^{2}(X)\right)$.

2.2. Quasi-locality. By definition, an operator in $\mathfrak{B}\left(\ell^{2}(X)\right)$ belongs to the uniform Roe algebra $C_{u}^{*}(X)$ if and only if it can be approximated by finite propagation operators in norm, which is usually not easy to check in practice. In order to find a more intrinsic and practical approach to characterise elements in $C_{u}^{*}(X)$, Roe introduced the following notion of quasi-locality.

Definition 2.1 ([23, 24]). Let $R, \varepsilon>0$. An operator $T \in \mathfrak{B}\left(\ell^{2}(X)\right)$ is said to have $(R, \varepsilon)$-propagation if for any $A, B \subseteq X$ such that $d(A, B) \geq R$, we have

$$
\left\|\chi_{A} T \chi_{B}\right\| \leq \varepsilon \text {. }
$$

We say that $T$ is quasi-local, if for all $\varepsilon>0$, there exists $R>0$ such that $T$ has $(R, \varepsilon)$-propagation.

It is routine to check that the set of all quasi-local operators in $\mathfrak{B}\left(\ell^{2}(X)\right)$ forms a $C^{*}$-subalgebra of $\mathfrak{B}\left(\ell^{2}(X)\right)$. Hence, we make the following definition:

Definition 2.2. Let $(X, d)$ be a discrete metric space. The uniform quasi-local algebra of $X$, denoted by $C_{u q}^{*}(X)$, is defined to be the $C^{*}$-algebra of all quasi-local operators in $\mathfrak{B}\left(\ell^{2}(X)\right)$.

It is clear that operators with finite propagation are quasi-local. Hence, $C_{u}^{*}(X) \subseteq$ $C_{u q}^{*}(X)$ after taking the closure.

2.3. Comparing $C_{u}^{*}(X)$ with $C_{u q}^{*}(X)$. We already noticed that $C_{u}^{*}(X) \subseteq C_{u q}^{*}(X)$ holds generally. For the opposite inclusion, the best existing result in this direction provides only a sufficient condition, which is Property A. Property A was introduced by $\mathrm{Yu}$ in [45] in his study of the coarse Baum-Connes conjecture and the Novikov conjecture. Here we recall some of equivalent characterisations of Property A and one of them is in terms of ghost operators: an operator $T \in \mathfrak{B}\left(\ell^{2}(X)\right)$ is called a ghost operator if for any $\varepsilon>0$, there exists a bounded subset $B \subseteq X$ such that for any $x, y \in X \backslash B$, we have $\left|T_{x, y}\right|<\varepsilon$. 
Proposition 2.3 ([38, Theorem 1.2.4], [26, Theorem 1.3]). Let $(X, d)$ be a metric space with bounded geometry. Then the following are equivalent:

(1) $(X, d)$ has Property $A$.

(2) For any $R, \varepsilon>0$ there exist a map $\xi: X \rightarrow \ell^{2}(X)$, and a number $S>0$ such that:

(a) $\left\|\xi_{x}\right\|_{2}=1$ for every $x \in X$;

(b) if $d(x, y)<R$, then $\left\|\xi_{x}-\xi_{y}\right\|_{2}<\varepsilon$;

(c) $\operatorname{supp}\left(\xi_{x}\right) \subseteq B(x, S)$ for every $x \in X$.

(3) All ghost operators in $C_{u}^{*}(X)$ are compact.

Finally, we recall the main result from [34] that $C_{u}^{*}(X)=C_{u q}^{*}(X)$ provided the space $X$ has Property A:

Proposition 2.4. [34, Theorem 3.3] Let $X$ be a metric space with bounded geometry. If $X$ has Property $A$, then $C_{u}^{*}(X)=C_{u q}^{*}(X)$.

\section{ASYMPTOTIC EXPANDERS}

In this section, we begin by recalling the notion of expander graphs and the averaging projection over a sequence of finite metric spaces. Then we introduce the notion of asymptotic expanders (see Definition A), which has close relation with the associated averaging projection and the uniform quasi-local algebra. Moreover, we show that being a sequence of asymptotic expanders is a coarse property under certain mild conditions (see Theorem 3.15 and Corollary 3.16).

By a graph in this paper we always mean an undirected graph with no loops and multiple edges, in the sense of graph theory. Let us fix further terminology: Let $X=(V, E)$ be a connected graph. The vertex set $V$ is regarded as a metric space equipped with the edge-path metric $d$. By a slight abuse of notation, this is the metric space we refer to when we regard $X$ as a metric space. We say that a graph $X$ has bounded valency if there exists some $k \in \mathbb{N}$ such that for any vertex $x \in V$, there are at most $k$ vertices connecting to $x$. It is clear that $X$ has bounded valency if and only if $(V, d)$ has bounded geometry. We set $\partial A:=\partial_{1} A$ to denote the 1-boundary of $A$.

3.1. Expander graphs. Recall that expander graphs are sequences of finite graphs which are highly connected but sparse at the same time. The first explicit construction was due to Margulis [19] using Kazhdan's property (T).

Definition 3.1. [20, Definition 5.6.2] Let $\left\{X_{n}=\left(V_{n}, E_{n}\right)\right\}_{n \in \mathbb{N}}$ be a sequence of finite graphs with bounded valency and $\left|V_{n}\right| \rightarrow \infty$ as $n \rightarrow \infty .\left\{X_{n}\right\}_{n \in \mathbb{N}}$ is said to be a sequence of expander graphs if there exists some $c>0$ such that for any $n \in \mathbb{N}$ and $A \subseteq V_{n}$ with $1 \leq|A| \leq\left|V_{n}\right| / 2$, then $|\partial A|>c|A|$.

Remark 3.2. The expanding condition implies that all the graphs $X_{n}$ in an expander sequence are connected (equivalently, 1-connected as metric spaces).

Alternatively, we have the following analytic characterisation of expander graphs (for a proof, see [9, Theorem 1.2.3], or additionally Section 5.6 of [20]):

Proposition 3.3. Let $X=\left\{X_{n}=\left(V_{n}, E_{n}\right)\right\}_{n \in \mathbb{N}}$ be a sequence of finite graphs with bounded valency and $\left|V_{n}\right| \rightarrow \infty$ as $n \rightarrow \infty$. Then $X$ is a sequence of expander graphs 
if and only if there exists some $c>0$ such that for any $n \in \mathbb{N}$ and any $f: V_{n} \rightarrow \mathbb{C}$ such that $\sum_{x \in V_{n}} f(x)=0$, the following Poincaré Inequality holds:

$$
\sum_{x, y \in V_{n} ; d(x, y)=1}|f(x)-f(y)|^{2} \geq c \sum_{x \in V_{n}}|f(x)|^{2} .
$$

Recall that the discrete Laplacian $\Delta_{Y}$ of a graph $Y=(V, E)$ is the $V$-by- $V$ matrix, with valencies of vertices on the diagonal; -1 at $(x, y)$-entry whenever there is an edge connecting $x$ and $y$; and 0 otherwise. For a sequence of graphs as in the proposition above, we denote by $\Delta$ the $X$-by- $X$ block-diagonal matrix with blocks being $\Delta_{X_{n}}$. This defines a bounded operator on $\ell^{2}(X)$ (because of the bounded valency) of propagation 1 .

A standard computation shows that the condition in Proposition 3.3 says that the discrete Laplacian $\Delta$ has a spectral gap, i.e., there exists $c>0$ such that $\sigma(\Delta) \subseteq$ $\{0\} \cup[c, \infty)$. Hence the characteristic function $\chi_{\{0\}}$ of the set $\{0\} \subset \mathbb{R}$ is continuous on the spectrum $\sigma(\Delta)$ of the operator $\Delta$. Thus we can apply continuous functional calculus, and obtain that $\chi_{\{0\}}(\Delta)$ is in the $C^{*}$-algebra generated by $\Delta$, which is contained in the uniform Roe algebra $C_{u}^{*}(X)$ (cf. discussion after [26, Proposition 3.1]). The operator $\chi_{\{0\}}(\Delta)$ is the projection onto the kernel of $\Delta$, which consists of functions constant on each $X_{n}$. This projection admits another description as the averaging projection $P_{X}$ on $X$, which is defined as follows:

Definition 3.4. Let $Y$ be a set and $F$ be a finite subset of $Y$. The averaging projection of $F$, denoted by $P_{F}$, is the orthogonal projection onto the span of $\chi_{F} \in$ $\ell^{2}(Y)$. In the matrix form, it can be represented by:

$$
\left(P_{F}\right)_{x, y}= \begin{cases}1 /|F|, & x, y \in F, \\ 0, & \text { otherwise. }\end{cases}
$$

Given a sequence of finite sets, let $X=\bigsqcup_{n \in \mathbb{N}} X_{n}$ as a set. Define the averaging projection of the sequence $\left\{X_{n}\right\}_{n \in \mathbb{N}}$ to be

$$
P_{X}:=\sum_{n \in \mathbb{N}} P_{X_{n}} \in \mathfrak{B}\left(\ell^{2}(X)\right),
$$

which converges in the strong operator topology on $\mathfrak{B}\left(\ell^{2}(X)\right)$. (We are indulging in a slight abuse of notation here, cf. Remark 3.5 below.)

Given a sequence of finite metric spaces $\left\{\left(X_{n}, d_{n}\right)\right\}_{n \in \mathbb{N}}$, we endow $X=\bigsqcup_{n \in \mathbb{N}} X_{n}$ with a metric: we say that $(X, d)$ is a coarse disjoint union of $\left\{X_{n}\right\}_{n \in \mathbb{N}}$, if the metric $d$ on each $X_{n}$ agrees with $d_{n}$ and satisfies:

$$
d\left(X_{n}, X_{m}\right) \rightarrow \infty \text { as } n+m \rightarrow \infty \text { and } m \neq n .
$$

Note that such a metric $d$ is well defined up to coarse equivalence. Whenever we need a concrete choice, we shall take $d$ such that for all $m, n \in \mathbb{N}, m \neq n$ and $x \in X_{n}, y \in X_{m}$ we have $d(x, y)=n+m+\operatorname{diam}\left(X_{n}\right)+\operatorname{diam}\left(X_{m}\right)$. For any such metric, $P_{X} \in \mathfrak{B}\left(\ell^{2}(X)\right)$ is a non-compact ghost projection.

Remark 3.5. The projection $P_{X}$ depends on the choice of the sequence $\left\{X_{n}\right\}_{n \in \mathbb{N}}$, not only on the (bijective) coarse equivalence type of a coarse disjoint union $X$ itself. For a simple example, consider the sequence with each $X_{n}=\left\{x_{n}\right\}$ consisting of a single point. First, we endow $X=\bigsqcup_{n \in \mathbb{N}} X_{n}$ with the metric as above; then $P_{X}=\mathrm{Id} \in$ $\mathfrak{B}\left(\ell^{2}(X)\right)$. However, if we denote $Y_{k}=\left\{x_{2 k-1}, x_{2 k}\right\}$ and declare $d\left(x_{2 k-1}, x_{2 k}\right)=k$ for 
$k \in \mathbb{N}$, then $Y=\bigsqcup_{k \in \mathbb{N}} Y_{k}$ is bijectively coarsely equivalent to $X$ (as sets, $X=Y$ ), but $P_{Y} \neq \mathrm{Id} \in \mathfrak{B}\left(\ell^{2}(X)\right)$.

From the discussion before Definition 3.4, we obtain the following:

Corollary 3.6. Let $\left\{X_{n}\right\}_{n \in \mathbb{N}}$ be a sequence of expander graphs, and let $X=\bigsqcup_{n \in \mathbb{N}} X_{n}$ be a coarse disjoint union. Then the averaging projection $P_{X}$ belongs to the uniform Roe algebra $C_{u}^{*}(X)$.

The following example is implicitly suggested in [35, Proposition 2.4] and it was brought to our attention by Sako. It shows that the converse does not hold in general.

Example 3.7. Let $X$ be the coarse disjoint union of a sequence of expander graphs $\left\{X_{n}\right\}_{n \in \mathbb{N}}$ with bounded valency at most $k$. For any $n \in \mathbb{N}$, choose an arbitrary finite graph $F_{n}$ of degree at most $k$, satisfying $\left|F_{n}\right| \rightarrow \infty$ and $\left|F_{n}\right| /\left|X_{n}\right| \rightarrow 0$ as $n \rightarrow \infty$. Here we regard $X_{n}$ and $F_{n}$ as metric spaces with the edge-path metrics.

For each $n \in \mathbb{N}$, we construct a new graph $Y_{n}$ which is the disjoint union of $X_{n}$ and $F_{n}$ except that one additional edge is attached between two chosen vertices $x_{n}$ in $X_{n}$ and $y_{n}$ in $F_{n}$. Clearly, $Y_{n}$ is a finite graph of valency at most $k+1$. We claim that $\left\{Y_{n}\right\}_{n \in \mathbb{N}}$ is not a sequence of expander graphs, but the averaging projection $P_{Y}$ belongs to the uniform Roe algebra $C_{u}^{*}(Y)$, where $Y$ is the coarse disjoint union of $\left\{Y_{n}\right\}_{n \in \mathbb{N}}$.

Indeed, since $\left|F_{n}\right| /\left|X_{n}\right| \rightarrow 0$, we can take a sufficiently large $n$ such that $\left|F_{n}\right| \leq$ $\left|Y_{n}\right| / 2$. By construction, $\partial F_{n}=\left\{x \in X_{n}: d\left(x, F_{n}\right)=1\right\}=\left\{x_{n}\right\}$, which implies that $\left|\partial F_{n}\right| /\left|F_{n}\right| \rightarrow 0$. Hence, $Y=\left\{Y_{n}\right\}_{n \in \mathbb{N}}$ is not a sequence of expander graphs.

Now we show that the averaging projection $P_{Y}$ belongs to the uniform Roe algebra $C_{u}^{*}(Y)$. In fact, this follows directly from [35, Proposition 2.4]. For convenience of the readers, we provide a proof here. Since $X$ is a subspace of $Y$, we have $P_{X} \in$ $\mathfrak{B}\left(\ell^{2}(X)\right) \subseteq \mathfrak{B}\left(\ell^{2}(Y)\right)$. We claim that the difference $P_{Y}-P_{X}$ is a compact operator in $\mathfrak{B}\left(\ell^{2}(Y)\right)$. In fact, a direct calculation shows that for each $n$ and $x, y \in Y_{n}$ :

$$
\left(P_{Y_{n}}-P_{X_{n}}\right)_{x, y}= \begin{cases}-\frac{\left|F_{n}\right|}{\left|X_{n}\right|\left(\left|X_{n}\right|+\left|F_{n}\right|\right)}, & x, y \in X_{n} \\ \frac{1}{\left|X_{n}\right|+\left|F_{n}\right|}, & \text { otherwise. }\end{cases}
$$

Since each operator $P_{Y_{n}}-P_{X_{n}}$ is represented by a finite matrix, its operator norm does not exceed its Frobenius norm:

$$
\begin{aligned}
\left\|P_{Y_{n}}-P_{X_{n}}\right\|_{F}^{2} & =\sum_{x, y \in Y_{n}}\left|\left(P_{Y_{n}}-P_{X_{n}}\right)_{x, y}\right|^{2} \\
& =\sum_{(x, y) \in X_{n}^{2}} \frac{\left|F_{n}\right|^{2}}{\left|X_{n}\right|^{2}\left(\left|X_{n}\right|+\left|F_{n}\right|\right)^{2}}+\sum_{(x, y) \in Y_{n}^{2} \backslash X_{n}^{2}} \frac{1}{\left(\left|X_{n}\right|+\left|F_{n}\right|\right)^{2}} \\
& =\frac{\left|X_{n}\right|^{2} \cdot\left|F_{n}\right|^{2}}{\left|X_{n}\right|^{2}\left(\left|X_{n}\right|+\left|F_{n}\right|\right)^{2}}+\frac{\left(\left|X_{n}\right|+\left|F_{n}\right|\right)^{2}-\left|X_{n}\right|^{2}}{\left(\left|X_{n}\right|+\left|F_{n}\right|\right)^{2}} \\
& =\frac{2\left|X_{n}\right| \cdot\left|F_{n}\right|+2\left|F_{n}\right|^{2}}{\left|X_{n}\right|^{2}+2\left|X_{n}\right| \cdot\left|F_{n}\right|+\left|F_{n}\right|^{2}} .
\end{aligned}
$$

By the assumption that $\left|F_{n}\right| /\left|X_{n}\right| \rightarrow 0$, we have $\left\|P_{Y_{n}}-P_{X_{n}}\right\| \rightarrow 0$ as $n \rightarrow \infty$. Hence $P_{Y}-P_{X}=\sum_{n \in \mathbb{N}}\left(P_{Y_{n}}-P_{X_{n}}\right)$ converges in the operator norm. Since each block $P_{Y_{n}}-P_{X_{n}}$ has finite rank, it is clear that $P_{Y}-P_{X}$ is a compact operator. From Corollary 3.6, $P_{X} \in C_{u}^{*}(X) \subseteq C_{u}^{*}(Y)$, which implies $P_{Y} \in C_{u}^{*}(Y)$ as required. 
3.2. Asymptotic expanders. Example 3.7 shows that the property of being a sequence of expander graphs cannot be characterised by the condition that the averaging projection belongs to the uniform Roe algebra. However, the counterexample is just a slight deformation of expanders.

In this section, we explore when the averaging projection $P_{X}$ is quasi-local, and introduce the notion of asymptotic expanders. We start with some elementary calculations.

Lemma 3.8. Let $X$ be a discrete metric space, $F$ be a finite subset of $X$ and $A, B \subseteq F$. Then

$$
\left\|\chi_{A} P_{F} \chi_{B}\right\|=\frac{\sqrt{|A||B|}}{|F|} .
$$

Proof. Without loss of generality, we may assume that $X=F$. By a direct calculation, we have

$$
\begin{aligned}
\left\|\chi_{A} P_{F} \chi_{B}\right\| & =\sup _{\|v\|=\|w\|=1}\left\langle\chi_{A} P_{F} \chi_{B} v, w\right\rangle=\sup _{\|v\|=\|w\|=1}\left\langle P_{F} \chi_{B} v, P_{F} \chi_{A} w\right\rangle \\
& =\sup _{\|v\|=\|w\|=1}\left\langle\sum_{f \in F}\left(\frac{1}{|F|} \sum_{a \in A} v(a)\right) \delta_{f}, \sum_{f \in F}\left(\frac{1}{|F|} \sum_{b \in B} w(b)\right) \delta_{f}\right\rangle \\
& =\sup _{\|v\|=\|w\|=1} \frac{1}{|F|^{2}} \cdot|F| \cdot\left(\sum_{a \in A} v(a)\right)\left(\overline{\sum_{b \in B} w(b)}\right) \\
& \leq \sup _{\|v\|=\|w\|=1} \frac{1}{|F|} \sqrt{|A|} \sqrt{|B|} \cdot\|v\| \cdot\|w\| \\
& \leq \frac{\sqrt{|A||B|}}{|F|}
\end{aligned}
$$

where the penultimate inequality follows from the Cauchy-Schwarz Inequality. On the other hand, it is easy to see that

$$
\left\langle P_{F} \chi_{B} v, P_{F} \chi_{A} w\right\rangle=\frac{\sqrt{|A||B|}}{|F|},
$$

where $v, w$ are the normalised characteristic functions of $A, B$, respectively.

From the definition of quasi-locality and the previous lemma, we directly obtain the following:

Proposition 3.9. Let $\left\{X_{n}\right\}_{n \in \mathbb{N}}$ be a sequence of finite metric spaces with $\left|X_{n}\right| \rightarrow \infty$ as $n \rightarrow \infty$. Let $X=\bigsqcup_{n \in \mathbb{N}} X_{n}$ be a coarse disjoint union, and let $P_{X}$ the averaging projection of the sequence $\left\{X_{n}\right\}_{n \in \mathbb{N}}$. Then $P_{X}$ is quasi-local if and only if

$$
0=\lim _{R \rightarrow+\infty} \sup \left\{\frac{|A||B|}{\left|X_{n}\right|^{2}}: n \in \mathbb{N}, A, B \subseteq X_{n}, d(A, B) \geq R\right\} .
$$

Remark 3.10. The limit above always exists, since the function

$$
R \mapsto \sup \left\{\frac{|A||B|}{\left|X_{n}\right|^{2}}: n \in \mathbb{N}, A, B \subseteq X_{n}, d(A, B) \geq R\right\}
$$

is non-increasing and bounded from below by 0 .

We now establish the geometric conditions equivalent to $P_{X}$ being quasi-local. 
Theorem 3.11. Let $\left\{X_{n}\right\}_{n \in \mathbb{N}}$ be a sequence of finite metric spaces with $\left|X_{n}\right| \rightarrow \infty$ as $n \rightarrow \infty$. Let $X=\bigsqcup_{n \in \mathbb{N}} X_{n}$ be a coarse disjoint union, and let $P_{X}$ the averaging projection of the sequence. Then the following are equivalent:

(1) $P_{X}$ is quasi-local;

(2) for any $\alpha \in\left(0, \frac{1}{2}\right]$, any $c \in(0,1)$, there exists $R>0$ such that for any $n \in \mathbb{N}$ and $A \subseteq X_{n}$ with $\alpha\left|X_{n}\right| \leq|A| \leq\left|X_{n}\right| / 2$, we have $\left|\partial_{R} A\right|>c|A|$;

(3) for any $\alpha \in\left(0, \frac{1}{2}\right]$, there exists $c \in(0,1)$ and $R>0$ such that for any $n \in \mathbb{N}$ and $A \subseteq X_{n}$ with $\alpha\left|X_{n}\right| \leq|A| \leq\left|X_{n}\right| / 2$, we have $\left|\partial_{R} A\right|>c|A|$.

Proof. "(1) $\Rightarrow$ (2)": Suppose (2) fails. Then there exists $\alpha_{0}>0$ and $c_{0} \in(0,1)$ such that for any $R>0$, there exists $n \in \mathbb{N}$ and $A_{n} \subseteq X_{n}$ with $\alpha_{0}\left|X_{n}\right| \leq\left|A_{n}\right| \leq\left|X_{n}\right| / 2$, while $\left|\partial_{R} A_{n}\right| \leq c_{0}\left|A_{n}\right|$. Now for any $R>0$, we have

$$
X_{n}=\left(X_{n} \backslash \mathcal{N}_{R}\left(A_{n}\right)\right) \sqcup A_{n} \sqcup \partial_{R} A_{n},
$$

which implies

$$
\begin{aligned}
\left|X_{n} \backslash \mathcal{N}_{R}\left(A_{n}\right)\right| & =\left|X_{n}\right|-\left|A_{n}\right|-\left|\partial_{R} A_{n}\right| \geq\left|X_{n}\right|-\left|A_{n}\right|-c_{0}\left|A_{n}\right|=\left|X_{n}\right|-\left(1+c_{0}\right)\left|A_{n}\right| \\
& \geq\left|X_{n}\right|-\frac{1+c_{0}}{2}\left|X_{n}\right|=\frac{1-c_{0}}{2}\left|X_{n}\right| .
\end{aligned}
$$

Hence we have

$$
\frac{\left|A_{n}\right| \cdot\left|X_{n} \backslash \mathcal{N}_{R}\left(A_{n}\right)\right|}{\left|X_{n}\right|^{2}} \geq \frac{\alpha_{0}\left|X_{n}\right| \cdot \frac{1-c_{0}}{2}\left|X_{n}\right|}{\left|X_{n}\right|^{2}}=\frac{\alpha_{0}\left(1-c_{0}\right)}{2}>0,
$$

which implies that the limit is bounded from below:

$$
\lim _{R \rightarrow+\infty} \sup \left\{\frac{|A||B|}{\left|X_{n}\right|^{2}}: n \in \mathbb{N}, A, B \subseteq X_{n}, d(A, B) \geq R\right\} \geq \frac{\alpha_{0}\left(1-c_{0}\right)}{2}>0 .
$$

This is a contradiction to the assumption that $P_{X}$ is quasi-local by Proposition 3.9.

"(2) $\Rightarrow(3) "$ : This is clear.

"(3) $\Rightarrow(1)$ ": Suppose $P_{X}$ is not quasi-local. Then by Proposition 3.9, we know that

$$
\alpha:=\frac{1}{2} \lim _{R \rightarrow+\infty} \sup \left\{\frac{|A||B|}{\left|X_{n}\right|^{2}}: n \in \mathbb{N}, A, B \subseteq X_{n}, d(A, B) \geq R\right\}>0 .
$$

Hence there exists an increasing sequence of natural numbers $\left\{m_{n}\right\}_{n \in \mathbb{N}}$ going to infinity, and $A_{n}, B_{n} \subseteq X_{m_{n}}$ with $d\left(A_{n}, B_{n}\right)>2 n$ such that $\left|A_{n}\right| \cdot\left|B_{n}\right| \geq \alpha \cdot\left|X_{m_{n}}\right|^{2}$. Since $\left|A_{n}\right| \leq\left|X_{m_{n}}\right|$ and $\left|B_{n}\right| \leq\left|X_{m_{n}}\right|$, we obtain that $\left|A_{n}\right| \geq \alpha\left|X_{m_{n}}\right|$ and $\left|B_{n}\right| \geq$ $\alpha\left|X_{m_{n}}\right|$.

By condition (3), for the above $\alpha$ there exists $c_{0} \in(0,1)$ and $R_{0}>0$ such that for any $n \in \mathbb{N}$ and $A^{\prime} \subseteq X_{n}$ with $\alpha\left|X_{n}\right| \leq\left|A^{\prime}\right| \leq\left|X_{n}\right| / 2$, we have $\left|\partial_{R_{0}} A^{\prime}\right|>c_{0}\left|A^{\prime}\right|$. Now consider $\mathcal{N}_{n}\left(A_{n}\right)$ and $\mathcal{N}_{n}\left(B_{n}\right)$. First, since $n \leq m_{n}$, we have $\mathcal{N}_{n}\left(A_{n}\right), \mathcal{N}_{n}\left(B_{n}\right) \subseteq X_{m_{n}}$. Since $d\left(A_{n}, B_{n}\right)>2 n$, we know that $\mathcal{N}_{n}\left(A_{n}\right)$ and $\mathcal{N}_{n}\left(B_{n}\right)$ are disjoint. Without loss of generality, we may assume that $\left|\mathcal{N}_{n}\left(A_{n}\right)\right| \leq\left|X_{m_{n}}\right| / 2$. Hence, we have

$$
\alpha\left|X_{m_{n}}\right| \leq\left|A_{n}\right| \leq\left|\mathcal{N}_{n}\left(A_{n}\right)\right| \leq\left|X_{m_{n}}\right| / 2 .
$$

By induction, we have

$$
\left|\mathcal{N}_{n}\left(A_{n}\right)\right| \geq\left(1+c_{0}\right)\left|\mathcal{N}_{n-R_{0}}\left(A_{n}\right)\right| \geq \ldots \geq\left(1+c_{0}\right)^{\left\lfloor n / R_{0}\right\rfloor}\left|A_{n}\right|
$$

for any $n \in \mathbb{N}$. Hence, we have

$$
\left|X_{m_{n}}\right| \geq\left|\mathcal{N}_{n}\left(A_{n}\right)\right| \geq\left(1+c_{0}\right)^{\left\lfloor n / R_{0}\right\rfloor}\left|A_{n}\right| \geq\left(1+c_{0}\right)^{\left\lfloor n / R_{0}\right\rfloor} \alpha\left|X_{m_{n}}\right|
$$


for any $n \in \mathbb{N}$, which is a contradiction.

It is clear from Definition 3.1 that for a sequence of expander graphs, condition (3) in the above proposition holds. Hence, we introduce the following notion:

Definition 3.12. 2 A sequence of finite metric spaces $\left\{X_{n}\right\}_{n \in \mathbb{N}}$ such that $\left|X_{n}\right| \rightarrow \infty$ (as $n \rightarrow \infty$ ) is said to be a sequence of asymptotic expanders (or asymptotic expander graphs when all $X_{n}$ are graphs) if for any $\alpha>0$, there exist $c \in(0,1)$ and $R>0$ such that for any $n \in \mathbb{N}$ and $A \subseteq X_{n}$ with $\alpha\left|X_{n}\right| \leq|A| \leq\left|X_{n}\right| / 2$, we have $\left|\partial_{R} A\right|>c|A|$.

By Example 3.7 and Theorem 3.11, there do exist asymptotic expander graphs which are not expander graphs:

Corollary 3.13. The sequence $\left\{Y_{n}\right\}_{n \in \mathbb{N}}$ constructed in Example 3.7 is a sequence of asymptotic expander graphs, but not expander graphs.

3.3. Coarse Invariance. In this subsection, we prove that being a sequence of asymptotic expanders is a coarse property, i.e. that it is invariant under coarse equivalence, provided that the equivalence respects the pieces. (Note that this is automatic when the terms of the sequence are sufficiently connected, e.g. when they are connected graphs.)

Note that "being a sequence of expander graphs" is preserved under coarse equivalences. This is well-known to experts and a detailed proof can be found, for example, in [31, Lemma 2.7.5] and [29, Lemma 12].

Remark 3.14. In general, "being a sequence of asymptotic expanders" is not a coarse property of the coarse disjoint union. Consider the following example: let $\left\{W_{n}\right\}_{n \in \mathbb{N}}$ be a sequence of expander graphs with $n /\left|W_{n}\right| \rightarrow 0$, and $\left\{Z_{n}\right\}_{n \in \mathbb{N}}$ be a sequence of Cayley graphs of $\mathbb{Z} / n \mathbb{Z}$ (with respect to the image of the generator $1 \in \mathbb{Z}$ ). Next, let $X_{n}:=W_{n} \sqcup Z_{n}$ for each $n \in \mathbb{N}$, where we declare the metric $d_{n}$ on $X_{n}$ to agree with the existing metrics on $W_{n}, Z_{n}$, and such that $d\left(W_{n}, Z_{n}\right)=2 n$. Let $Y_{2 k}:=W_{k}$ and $Y_{2 k-1}:=Z_{k}$ for $k \in \mathbb{N}$.

Now consider coarse disjoint unions $X=\bigsqcup_{n \in \mathbb{N}} X_{n}$ and $Y=\bigsqcup_{n \in \mathbb{N}} Y_{n}$. Then $X$ and $Y$ are (bijectively) coarsely equivalent (essentially via assembling the identity maps on each $W_{n}$ and $Z_{n}$ ). However the sequence $\left\{X_{n}\right\}_{n \in \mathbb{N}}$ is a sequence of asymptotic expanders (this follows from a computation analogous to Example 3.7), but $\left\{Y_{n}\right\}_{n \in \mathbb{N}}$ is not. Indeed, the condition (2) of Theorem 3.11 fails: Let $\alpha=\frac{1}{2}$ and $c=\frac{1}{2}$. Given $R \geq 1$, take $n=8 R$, we choose the subset $A_{n} \subset Y_{2 n-1}=\mathbb{Z} / n \mathbb{Z}$ to be the image of $\{1,2, \ldots, 4 R\} \subset \mathbb{Z}$. Then $\left|A_{n}\right|=\left|Y_{2 n-1}\right| / 2=\alpha\left|Y_{2 n-1}\right|$ and $\left|\partial_{R} A_{n}\right|=2 R=c\left|A_{n}\right|$.

The issue here is that the coarse equivalence between $X$ and $Y$ does not map "pieces to pieces". This is explicitly prevented in the following theorem. Another way to circumvent this issue is to assume that the pieces are sufficiently connected, e.g. connected graphs (see Corollary 3.16).

Theorem 3.15. Let $\left\{X_{n}\right\}_{n \in \mathbb{N}}$ and $\left\{Y_{n}\right\}_{n \in \mathbb{N}}$ be sequences of finite metric spaces, such that $\left|X_{n}\right|,\left|Y_{n}\right| \rightarrow \infty$ as $n \rightarrow \infty$, and coarse disjoint unions $X=\bigsqcup_{n \in \mathbb{N}} X_{n}$ and $Y=\bigsqcup_{n \in \mathbb{N}} Y_{n}$ have bounded geometry. Let $\varphi_{n}: X_{n} \rightarrow Y_{n}$, for $n \in \mathbb{N}$, be functions such that $\varphi=\bigsqcup_{n \in \mathbb{N}} \varphi_{n}: X \rightarrow Y$ is a coarse equivalence. Then if $\left\{X_{n}\right\}_{n \in \mathbb{N}}$ is a sequence of asymptotic expanders, then so is $\left\{Y_{n}\right\}_{n \in \mathbb{N}}$.

\footnotetext{
${ }^{2}$ After we have already finished the paper, we were informed by R. Grigorchuk that a different notion of asymptotic expanders was introduced by him [12, Definition 10.3]. As far as we see, these two notions are not directly related to each other.
} 
Before embarking on the proof of Theorem 3.15, let us point out that under a suitable connectedness assumption, we can simplify the statement:

Corollary 3.16. Let $\left\{X_{n}\right\}_{n \in \mathbb{N}}$ and $\left\{Y_{n}\right\}_{n \in \mathbb{N}}$ be sequences of finite metric spaces, such that $\left|X_{n}\right|,\left|Y_{n}\right| \rightarrow \infty$ as $n \rightarrow \infty$, and coarse disjoint unions $X=\bigsqcup_{n \in \mathbb{N}} X_{n}$ and $Y=\bigsqcup_{n \in \mathbb{N}} Y_{n}$ have bounded geometry. Assume that there exists a $D \geq 0$, such that $X_{n}$ and $Y_{n}$ are $D$-connected for each $n \in \mathbb{N}$. Then if $X$ and $Y$ are coarsely equivalent, and $\left\{X_{n}\right\}_{n \in \mathbb{N}}$ is a sequence of asymptotic expanders, then so is $\left\{Y_{n}\right\}_{n \in \mathbb{N}}$.

Proof of Corollary [3.16. Let $f: X \rightarrow Y$ be a coarse equivalence and denote $f_{n}=$ $\left.f\right|_{X_{n}}$. Then by the proof of [16, Lemma 1], $f$ builds up a bijection between a co-finite subset of $\left\{X_{n}\right\}_{n \in \mathbb{N}}$ and a co-finite subset of $\left\{Y_{n}\right\}_{n \in \mathbb{N}}$. (Note that [16 assumes that the pieces $X_{n}$ and $Y_{n}$ are connected graphs; however the argument for the above claim carries through with obvious changes when the pieces are assumed to be $D$ connected.) Consequently, there exists $N \in \mathbb{N}$, such that for all $n \geq N$ there exists $k(n) \in \mathbb{N}$, such that $f_{n}: X_{n} \rightarrow Y_{k(n)}$, and $\mathbb{N} \backslash\{k(n): n \geq N\}$ is finite.

Now $\left\{X_{n}\right\}_{n \in \mathbb{N}}$ is a sequence of asymptotic expanders if and only if $\left\{X_{n}\right\}_{n \geq N}$ is, and likewise for $\left\{Y_{n}\right\}_{n \in \mathbb{N}}$ and $\left\{Y_{k(n)}\right\}_{n \geq \mathbb{N}}$. The proof is finished by applying Theorem 3.15 to the latter two sequences.

We now turn to the proof of Theorem 3.15, which will be split into several lemmas. First, we fix some notation. We shall use $\partial_{R}^{\text {in }}(A)=\left\{x \in A: d\left(x, A^{c}\right) \leq R\right\}$ for the inner $R$-boundary of a set $A \subset X$. We shall say that a subset $S \subset X$ is $D$-dense in $X$ (for some $D \geq 0$ ), if any point of $X$ is within distance $D$ of some point in $S$. Finally, recall that if $X$ has bounded geometry, we denote $N_{X}(R)=\sup _{x \in X}|B(x, R)|$.

Lemma 3.17. Let $\psi: X \rightarrow Y$ be a function between two finite metric spaces, such that $\psi(X)$ is $D$-dense in $Y$ for some $D \geq 0$. Let $B \subseteq Y$. Then

$$
\left|\psi^{-1}(B)\right| \geq|B| \cdot \frac{1}{N_{Y}(D)} \cdot\left(1-\frac{\left|\partial_{D}^{\text {in }}(B)\right|}{|B|}\right) .
$$

Proof. Define $I:=\{y \in B: B(y, D) \subseteq B\}$. Given $y \in I$, there exists $x_{y} \in X$ such that $d\left(\psi\left(x_{y}\right), y\right) \leq D$, because $\psi(X)$ is $D$-dense in $Y$. Hence $\psi\left(x_{y}\right) \in B$, so $x_{y} \in \psi^{-1}(B)$. This defines an assignment $I \ni y \mapsto x_{y} \in \psi^{-1}(B)$, with at most $N_{Y}(D)$ elements of $I$ mapping to the same $x_{y} \in \psi^{-1}(B)$ (the points in $B\left(\psi\left(x_{y}\right), D\right)$ ). Hence $\left|\psi^{-1}(B)\right| \geq|I| / N_{Y}(D)$.

Next, if $y \in B \backslash I$, then $y \in \partial_{D}^{\text {in }}(B)$, so $|I| \geq|B|-\left|\partial_{D}^{\text {in }}(B)\right|$. Combining the two inequalities yields the desired one.

Lemma 3.18. Under the assumptions of Lemma 3.17, assume further that $|B| \leq$ $|Y| / 2$, and that $1-N_{Y}(D) \frac{\left|\partial_{D}(B)\right|}{|B|} \geq \frac{1}{2}$. Then either $A=\psi^{-1}(B)$ or $A=\psi^{-1}(Y \backslash B)$ satisfies $|A| \leq|X| / 2$ and $|A| \geq \frac{1}{2 N_{Y}(D)}|B|$.

Proof. Suppose that $\left|\psi^{-1}(B)\right| \leq|X| / 2$. We apply Lemma 3.17 with $B$ and let $A:=\psi^{-1}(B)$. Inserting the general observation $\left|\partial_{D}^{\text {in }}(B)\right| \leq N_{Y}(D)\left|\partial_{D}(B)\right|$ into (3.2) and applying the assumption immediately yield $|A| \geq \frac{1}{2 N_{Y}(D)}|B|$.

On the other hand, if $\left|\psi^{-1}(B)\right|>|X| / 2$, we let $A:=\psi^{-1}(Y \backslash B)=X \backslash \psi^{-1}(B)$ (as then $|A| \leq|X| / 2)$. Note that as $|B| \leq|Y| / 2$, we have $|Y \backslash B| \geq|B|$. Furthermore, note that $1-\frac{\left|\partial_{D}(B)\right|}{|B|} \geq 1-N_{Y}(D) \frac{\left|\partial_{D}(B)\right|}{|B|} \geq \frac{1}{2}$. 
We apply Lemma 3.17 with $Y \backslash B$ in place of $B$. From (3.2) we then get

$$
\begin{aligned}
|A| & =\left|\psi^{-1}(Y \backslash B)\right| \geq|Y \backslash B| \cdot \frac{1}{N_{Y}(D)} \cdot\left(1-\frac{\left|\partial_{D}^{\text {in }}(Y \backslash B)\right|}{|Y \backslash B|}\right) \\
& \geq|B| \cdot \frac{1}{N_{Y}(D)} \cdot\left(1-\frac{\left|\partial_{D}(B)\right|}{|B|}\right) \geq \frac{1}{2 N_{Y}(D)}|B|,
\end{aligned}
$$

which finishes the proof.

Lemma 3.19. With the notation as in Lemma 3.17, assume further that $\psi$ is at most $K$-to-one (for some $K \geq 1$ ). Furthermore, let $\rho_{+}$be any function which for all $u, v \in X$ satisfies $d(\psi(u), \psi(v)) \leq \rho_{+}(d(u, v))$. Then for any $S \geq 0$ both $A=\psi^{-1}(B)$ and $A=\psi^{-1}(Y \backslash B)=X \backslash \psi^{-1}(B)$ satisfy $\left|\partial_{S}(A)\right| \leq K N_{X}(S)\left|\partial_{\rho_{+}(S)}(B)\right|$.

Proof. Denote $C=\psi^{-1}(B)$. Let $u \in \partial_{S}(C)$. Then there is a $v \in C$ with $d(u, v) \leq S$. Hence $\psi(v) \in B, \psi(u) \notin B$ and $d(\psi(u), \psi(v)) \leq \rho_{+}(S)$. In other words, $\psi(u) \in$ $\partial_{\rho_{+}(S)}(B)$ and as $\psi$ is at most $K$-to-one, we get $\left|\partial_{S}(C)\right| \leq K\left|\partial_{\rho_{+}(S)}(B)\right|$.

When $A=\psi^{-1}(B)=C$, then the above inequality trivially implies $\left|\partial_{S}(A)\right| \leq$ $K N_{X}(S)\left|\partial_{\rho_{+}(S)}(B)\right|$.

When $A=\psi^{-1}(Y \backslash B)=X \backslash C$, we use the general fact that $\left|\partial_{S}(X \backslash C)\right| \leq$ $N_{X}(S)\left|\partial_{S}(C)\right|$. Now the inequality established in the first paragraph yields $\left|\partial_{S}(A)\right| \leq$ $N_{X}(S)\left|\partial_{X}(C)\right| \leq K N_{X}(A)\left|\partial_{\rho_{+}(S)}(B)\right|$.

Proof of Theorem 3.15. Recall that we have, for each $n \in \mathbb{N}$, a function $\varphi_{n}: X_{n} \rightarrow$ $Y_{n}$, such that $\varphi=\bigsqcup_{n \in \mathbb{N}} \varphi_{n}$ is a coarse equivalence between $X=\bigsqcup_{n \in \mathbb{N}} X_{n}$ and $Y=\bigsqcup_{n \in \mathbb{N}} Y_{n}$. Since both have bounded geometry, the functions $N_{X}$ and $N_{Y}$ work in particular for any $X_{n}$ or $Y_{n}$, respectively. As $\varphi$ is a coarse equivalence, there exist functions $\rho_{ \pm}: \mathbb{R}^{+} \rightarrow \mathbb{R}^{+}$such that $\rho_{-}(t) \rightarrow \infty$ as $t \rightarrow \infty$ and

$$
\rho_{-}(d(x, y)) \leq d\left(\varphi_{n}(x), \varphi_{n}(y)\right) \leq \rho_{+}(d(x, y))
$$

for any $n \in \mathbb{N}$ and $x, y \in X_{n}$. Without loss of generality, we may assume that both $\rho_{+}$and $\rho_{-}$are non-decreasing. Moreover, there exists a $D \geq 0$ such that $\varphi_{n}\left(X_{n}\right)$ is $D$-dense in $Y_{n}$. As $X$ has bounded geometry, it follows that there exists $K>0$ such that $\left|\varphi^{-1}(y)\right| \leq K$ for any $y \in Y$.

Assume that $\left\{Y_{n}\right\}$ is not a sequence of asymptotic expanders, i.e. there exists some $\alpha \in\left(0, \frac{1}{2}\right]$ such that for any $R>0$, there exist sequences $\left\{k_{n}\right\}_{n \in \mathbb{N}}$ and $\left\{B_{n}\right\}_{n \in \mathbb{N}}$ with $B_{n} \subseteq Y_{k_{n}}$ and $\alpha\left|Y_{k_{n}}\right| \leq\left|B_{n}\right| \leq\left|Y_{k_{n}}\right| / 2$, such that $\left|\partial_{R} B_{n}\right| /\left|B_{n}\right| \rightarrow 0$.

Given any $S \geq 0$, take $R>\max \left\{\rho_{+}(S), D\right\}$ and let $\left\{B_{n}\right\}_{n \in \mathbb{N}}$ be as described above. Without loss of generality, we can assume that $k_{n}=n$. Then $\partial_{D}\left(B_{n}\right) \subseteq$ $\partial_{R}\left(B_{n}\right)$, and so also $\left|\partial_{D}\left(B_{n}\right)\right| /\left|B_{n}\right| \rightarrow 0$; likewise $\left|\partial_{\rho_{+}(S)}\left(B_{n}\right)\right| \leq\left|\partial_{R}\left(B_{n}\right)\right|$. Thus for sufficiently large $n$ the assumptions of Lemma 3.18 are satisfied by $\varphi_{n}: X_{n} \rightarrow Y_{n}$ and $B_{n}$, so we get a sequence of subsets $A_{n} \subseteq X_{n}$ with $\frac{1}{2 N_{Y}(D)}\left|B_{n}\right| \leq\left|A_{n}\right| \leq\left|X_{n}\right| / 2$. By Lemma 3.19, they also satisfy $\left|\partial_{S}\left(A_{n}\right)\right| \leq K N_{X}(S)\left|\partial_{R}\left(B_{n}\right)\right|$.

Since $\varphi_{n}$ is at most $K$-to-one, we get

$$
\left|A_{n}\right| \geq \frac{1}{2 N_{Y}(D)}\left|B_{n}\right| \geq \frac{\alpha}{2 N_{Y}(D)}\left|Y_{n}\right| \geq \frac{\alpha}{2 K N_{Y}(D)}\left|X_{n}\right|
$$

so the cardinalities of $A_{n}$ are at least a uniform proportion of $X_{n}$. Finally,

$$
\frac{\left|\partial_{S}\left(A_{n}\right)\right|}{\left|A_{n}\right|} \leq \frac{K N_{X}(S)\left|\partial_{R}\left(B_{n}\right)\right|}{\frac{1}{2 N_{Y}(D)}\left|B_{n}\right|} \rightarrow 0 .
$$


Thus we have shown that $\left\{X_{n}\right\}$ is not a sequence of asymptotic expanders either.

\section{ASYMPTOTIC EXPANDER GRAPHS ARE NOT UNIFORMLY LOCALLY AMENABLE}

In this section, we show that being a sequence of asymptotic expanders leads to the failure of uniform local amenability. Recall from [3, Proposition 3.2] that Property A implies uniform local amenability, while the property of coarse embeddability into Hilbert spaces does not imply it generally (see [3, Corollary 4.3]). First let us recall the definition:

Definition 4.1 ([3, Definition 2.2]). A metric space $(X, d)$ is said to be uniformly locally amenable (ULA) if for all $R, \varepsilon>0$ there exists $S>0$ such that for any finite subset $F$ of $X$, there exists $E \subseteq X$ with $\operatorname{diam}(E) \leq S$ and $\left|\partial_{R}(E) \cap F\right|<\varepsilon|E \cap F|$.

Note that replacing $E$ with $E \cap F$, we can assume that $E \subseteq F$ in the above definition. We want to use another equivalent form of ULA as follows. For a finite subset $F \subseteq X$, define the associated normalised characteristic measure $\mu_{F}$ to be

$$
\mu_{F}(E):=\frac{|E \cap F|}{|F|}
$$

for any $E \subseteq X$. Clearly, $\mu_{F}$ is a probability measure with finite support $F$. Then we can translate ULA in the following language directly:

Lemma 4.2. A metric space $(X, d)$ is uniformly locally amenable if and only if for all $R, \varepsilon>0$ there exists $S>0$ such that for any finite $F \subseteq X$, there exists $E \subseteq F$ with $\operatorname{diam}(E) \leq S$ and $\mu_{F}\left(\partial_{R} E\right)<\mu_{F}(E)$.

By the same argument as in [3, Theorem 3.8], ULA implies a weaker version of the metric sparsification property introduced by Chen, Tessera, Wang and Yu [6] as follows:

Lemma 4.3. Let $(X, d)$ be a metric space with $U L A$. Then for any $c \in(0,1)$ and $R>0$, there exists $S>0$ such that for any finite $F \subseteq X$, there exists $\Omega \subseteq F$ with a decomposition $\Omega=\bigsqcup_{i \in I} \Omega_{i}$ satisfying the following:

- $\mu_{F}(\Omega) \geq c$;

- $\operatorname{diam}\left(\Omega_{i}\right) \leq S$;

- $d\left(\Omega_{i}, \Omega_{j}\right)>R$ for $i \neq j$.

Proof. Given $c \in(0,1)$ and $R>0$, take $\varepsilon=1 / c-1$. By Lemma 4.2, there exists $S>0$ satisfying the condition therein. Given a finite subset $F \subseteq X$, we set $F_{1}:=F$. By assumption, there exists $E_{1} \subseteq F_{1}$ with $\operatorname{diam}\left(E_{1}\right) \leq S$ and $\mu_{F}\left(\partial_{R} E_{1}\right)<\varepsilon \mu_{F}\left(E_{1}\right)$.

Now set $F_{2}:=F_{1} \backslash \mathcal{N}_{R}\left(E_{1}\right)$. By assumption, there exists $E_{2} \subseteq F_{2}$ with $\operatorname{diam}\left(E_{2}\right) \leq$ $S$ and $\mu_{F_{2}}\left(\partial_{R} E_{2}\right)<\varepsilon \mu_{F_{2}}\left(E_{2}\right)$. Hence $\left|\partial_{R} E_{2} \cap F_{2}\right|<\varepsilon\left|E_{2}\right|$, which implies $\mu_{F}\left(\partial_{R} E_{2} \cap\right.$ $\left.F_{2}\right)<\varepsilon \mu_{F}\left(E_{2}\right)$ since $F_{2} \subseteq F$.

Similarly, we may set $F_{3}:=F_{2} \backslash \mathcal{N}_{R}\left(E_{2}\right)$ and continue the process. Since $F_{1}$ is finite, it must eventually terminate, providing two sequences $F_{1} \supseteq F_{2} \supseteq \ldots \supseteq F_{n}$ and $E_{1}, E_{2}, \ldots, E_{n}$ such that $E_{i} \subseteq F_{i}$ for all $i$ and

- $\operatorname{diam}\left(E_{i}\right) \leq S$ for all $i$;

- $d\left(E_{i}, E_{j}\right)>R$ for $i \neq j$;

- $\mu_{F}\left(\partial_{R} E_{i} \cap F_{i}\right)<\varepsilon \mu_{F}\left(E_{i}\right)$. 
Set $\Omega_{i}:=E_{i}$ and $\Omega:=\bigsqcup_{1 \leq i \leq n} \Omega_{i}$. We have

$$
1=\mu_{F}\left(F_{1}\right)=\sum_{i=1}^{n} \mu_{F}\left(E_{i}\right)+\mu_{F}\left(\partial_{R} E_{i} \cap F_{i}\right)<\sum_{i=1}^{n}(1+\varepsilon) \mu_{F}\left(E_{i}\right)=(1+\varepsilon) \mu_{F}(\Omega),
$$

which implies that $\mu_{F}(\Omega)>1 /(1+\varepsilon)=c$. So we finish the proof.

Theorem 4.4. Let $X$ be a metric space with bounded geometry, which is a coarse disjoint union of a sequence of asymptotic expanders. Then $X$ is not uniformly locally amenable. In particular, $X$ does not have Property $A$.

Proof. By assumption, we can write $X=\bigsqcup_{n \in \mathbb{N}} X_{n}$, where $\left\{X_{n}\right\}_{n \in \mathbb{N}}$ is a sequence of asymptotic expanders. Assume that $X$ is uniformly locally amenable. Setting $c=1 / 2$ and given $R>0$, by Lemma 4.3 there exists $S=S(R)>0$ such that for any finite subset $F \subseteq X$, there exists $\Omega \subseteq F$ with a decomposition $\Omega=\bigsqcup_{i \in I} \Omega_{i}$ satisfying the conditions therein. Hence for each $n \in \mathbb{N}$, there exists $\Omega^{(n)} \subseteq X_{n}$ with a decomposition $\Omega^{(n)}=\bigsqcup_{i \in I} \Omega_{i}^{(n)}$ satisfying:

- $\left|\Omega^{(n)}\right| \geq\left|X_{n}\right| / 2$;

- $\operatorname{diam}\left(\Omega_{i}^{(n)}\right) \leq S(R)$;

- $d\left(\Omega_{i}^{(n)}, \Omega_{j}^{(n)}\right)>R$ for $i \neq j$.

As $X$ has bounded geometry, $N_{X}(S)=\sup _{x \in X}|B(x, S)|$ is finite. Since each $\Omega_{i}^{(n)}$ has cardinality at most $N_{X}(S)$, for any $n \in \mathbb{N}$ we may take a decomposition $I=I_{1} \sqcup I_{2}$ such that for

$$
A_{n}:=\bigsqcup_{i \in I_{1}} \Omega_{i}^{(n)} \subseteq X_{n} \quad \text { and } \quad B_{n}:=\bigsqcup_{i \in I_{2}} \Omega_{i}^{(n)} \subseteq X_{n}
$$

we have $A_{n} \sqcup B_{n}=\Omega^{(n)}$ and

$$
\left|A_{n}\right|,\left|B_{n}\right| \in\left[\frac{\left|\Omega^{(n)}\right|}{2}-N_{X}(S), \frac{\left|\Omega^{(n)}\right|}{2}+N_{X}(S)\right] .
$$

Note that by construction we have $d\left(A_{n}, B_{n}\right) \geq R$. For the given $R$ (and thus also $S=S(R)$ ), we may choose $n$ sufficiently large such that $\frac{N_{X}(S)}{\left|X_{n}\right|}<\frac{1}{32}$. Consequently

$$
\frac{\left|A_{n}\right| \cdot\left|B_{n}\right|}{\left|X_{n}\right|^{2}} \geq \frac{\left(\left|\Omega^{(n)}\right| / 2-N_{X}(S)\right)^{2}}{\left|X_{n}\right|^{2}} \geq \frac{\left|\Omega^{(n)}\right|^{2}}{4\left|X_{n}\right|^{2}}-\frac{\left|\Omega^{(n)}\right| \cdot N_{X}(S)}{\left|X_{n}\right|^{2}} \geq \frac{1}{16}-\frac{N_{X}(S)}{\left|X_{n}\right|} \geq \frac{1}{32} \text {. }
$$

In conclusion, for any $R>0$, we obtain $n \in \mathbb{N}$ and sets $A_{n}, B_{n} \subseteq X_{n}$, such that $d\left(A_{n}, B_{n}\right) \geq R$ and $\frac{\left|A_{n}\right| \cdot\left|B_{n}\right|}{\left|X_{n}\right|^{2}} \geq \frac{1}{32}$. This is a contradiction with the condition in Proposition 3.9, so $X$ is not uniformly locally amenable. Finally recall from 3 , Proposition 3.2] that Property A implies uniform local amenability, so $X$ does not have Property A and we finish the proof.

\section{NUClEARITY OF UNIFORM QUASI-LOCAL ALGEBRAS}

From [30, Theorem 5.3] and Proposition 2.4 we know that the uniform quasilocal algebra $C_{u q}^{*}(X)$ is nuclear for every metric space with bounded geometry and Property A. In this section, we provide a proof for the converse implication: the nuclearity of the uniform quasi-local algebra $C_{u q}^{*}(X)$ implies that $X$ has Property A.

Firstly, let us recall some related notions and facts: 
Definition 5.1 ([5, Definition 2.1.1 and Definition 2.3.1]). Let $\mathcal{A}$ and $\mathcal{B}$ be two $C^{*}$ algebras. A map $\theta: \mathcal{A} \rightarrow \mathcal{B}$ is called nuclear if for any $\varepsilon>0$ and any finite subset $F \subseteq \mathcal{A}$, there exist $n \in \mathbb{N}$ and contractive completely positive maps $\varphi: \mathcal{A} \rightarrow M_{n}(\mathbb{C})$ and $\psi: M_{n}(\mathbb{C}) \rightarrow \mathcal{B}$ such that $\|\psi \circ \varphi(a)-\theta(a)\|<\varepsilon$ for any $a \in F$. A $C^{*}$-algebra $\mathcal{A}$ is called nuclear if the identity map $\operatorname{Id}_{\mathcal{A}}$ is nuclear.

Proposition 5.2 ([30, Theorem 5.3]). Let $(X, d)$ be a metric space with bounded geometry, then $X$ has Property $A$ if and only if the uniform Roe algebra $C_{u}^{*}(X)$ is nuclear.

We need the following auxiliary lemma characterising Property A, which is a slight modification of Proposition 2.3. The proof is elementary, hence we leave it to the readers.

Lemma 5.3. Let $(X, d)$ be a metric space with bounded geometry. Then the following are equivalent:

(1) $(X, d)$ has Property A.

(2) For any $R, \varepsilon>0$ there exist a map $\eta: X \rightarrow \ell^{2}(X)$ satisfying:

(a) $\left\|\eta_{x}\right\|_{2}=1$ for every $x \in X$;

(b) for $x, y \in X$ with $d(x, y)<R$, we have $\left\|\eta_{x}-\eta_{y}\right\|_{2}<\varepsilon$;

(c) $\lim _{S \rightarrow \infty} \sup _{x \in X} \sum_{z \notin B(x, S)}\left|\eta_{x}(z)\right|^{2}=0$.

Recall that an operator $T \in \mathfrak{B}\left(\ell^{2}(X)\right)$ is called a ghost operator if for any $\varepsilon>0$, there exists a bounded subset $B \subseteq X$ such that for any $x, y \in X \backslash B$, we have $\left|T_{x, y}\right|<\varepsilon$. It is easy to check that all the ghost operators in the uniform Roe algebra $C_{u}^{*}(X)$ form an ideal in $C_{u}^{*}(X)$. The same situation also holds in the case of uniform quasi-local algebra $C_{u q}^{*}(X)$ :

Lemma 5.4. All the ghost operators in the uniform quasi-local algebra $C_{u q}^{*}(X)$ form a two-sided closed ideal in $C_{u q}^{*}(X)$.

Proof. Let $G, T \in C_{u q}^{*}(X)$ with norm 1 and suppose $G$ is ghost. It suffices to show that $G T$ and $T G$ are ghost as well. We only prove the case of $G T$, while the case of $T G$ is similar.

Fix an $\varepsilon>0$. Since $T$ is quasi-local, there exists a $R>0$ such that for any $A, B \subseteq X$ with $d(A, B)>R$, then $\left\|\chi_{A} T \chi_{B}\right\|<\varepsilon$. Hence for any $y \in X$, we have

$$
\left\|\chi_{B(y, R)^{c}} T \chi_{\{y\}}\right\|=\left(\sum_{z: d(z, y)>R}\left|T_{z, y}\right|^{2}\right)^{\frac{1}{2}}<\varepsilon .
$$

Taking $\varepsilon^{\prime}:=\frac{\varepsilon}{N_{X}(R)}$, there exists a finite subset $B$ such that for any $x^{\prime}, y^{\prime} \in X \backslash B$, then $\left|G_{x^{\prime}, y^{\prime}}\right|<\varepsilon^{\prime}$. Now taking $B^{\prime}:=\mathcal{N}_{R}(B)$, and note that for any $y \notin B^{\prime}$ and $z \in B(y, R)$, we have $z \notin B$. Hence for any $x, y \in X \backslash B^{\prime}$, we have:

$$
\begin{aligned}
\left|(G T)_{x, y}\right| & \leq\left|\sum_{z: d(z, y) \leq R} G_{x, z} T_{z, y}\right|+\left|\sum_{z: d(z, y)>R} G_{x, z} T_{z, y}\right| \\
& \leq\left(\sum_{z: d(z, y) \leq R} \varepsilon^{\prime} \cdot\left|T_{z, y}\right|\right)+\|G\| \cdot\left(\sum_{z: d(z, y)>R}\left|T_{z, y}\right|^{2}\right)^{\frac{1}{2}} \\
& \leq \varepsilon^{\prime} \cdot|B(y, R)| \cdot\|T\|+\|G\| \cdot \varepsilon \\
& \leq 2 \varepsilon
\end{aligned}
$$


where we use the Cauchy-Schwartz Inequality in the second inequality. Since the closeness is clear, we finish the proof.

Now we are in the position to prove the following main result of this section, whose proof is inspired by that of [5, Theorem 5.5.7].

Theorem 5.5. For a metric space $(X, d)$ with bounded geometry, the following are equivalent:

(1) X has Property A;

(2) the uniform quasi-local algebra $C_{u q}^{*}(X)$ is nuclear;

(3) the canonical inclusion $C_{u}^{*}(X) \hookrightarrow C_{u q}^{*}(X)$ is nuclear;

(4) all ghost operators in the uniform quasi-local algebra $C_{u q}^{*}(X)$ are compact.

(5) $\ell^{\infty}(X)$ separates ideals of $C_{u q}^{*}(X)$. In other words, the closed ideal generated by $I \cap \ell^{\infty}(X)$ inside $C_{u q}^{*}(X)$ is equal to I for every closed ideal I in $C_{u q}^{*}(X)$.

Proof. From Proposition 2.4, we know that if $X$ has Property A then $C_{u}^{*}(X)=$ $C_{u q}^{*}(X)$. Hence combining with Proposition 5.2 , we have "(1) $\Rightarrow(2)$ "; and combining with Proposition 2.3, we have " $(1) \Rightarrow(4)$ ". It follows directly from [2, Theorem 3.20] (see also [7) and Lemma 5.4 that "(1) $\Rightarrow(5) \Rightarrow(4) "$ holds.

On the other hand, since $C_{u}^{*}(X)$ is a subalgebra of $C_{u q}^{*}(X)$, we know that condition (4) implies that all ghost operators in $C_{u}^{*}(X)$ are compact. Hence from Proposition 2.3 again, we obtain "(4) $\Rightarrow(1)$ ". Also notice that due to the fact that the composition of two completely positive maps is nuclear provided either one of them is, we know that " $(2) \Rightarrow(3)$ " holds.

Therefore, it suffices to prove "(3) $\Rightarrow(1)$ ". To summarise the rest of the proof, we follow [5, Theorem 5.5.7] to construct "Property A" vectors (Proposition 2.3); but in the last step, instead of uniform bound on supports, we use quasi-locality to get strong summability, as in condition (c) in Lemma 5.3.

Assume that the inclusion $C_{u}^{*}(X) \hookrightarrow C_{u q}^{*}(X)$ is nuclear. Let $R>0$ and $\varepsilon>0$. Since $X$ has bounded geometry, there exists a finite set $\mathcal{F}$ of partial isometries in $\mathbb{C}_{u}[X]$ with the property that for any $x, y \in X$ with $d(x, y) \leq R$, there exists $v \in \mathcal{F}$ such that $v \delta_{x}=\delta_{y}$ (see e.g. [34, Lemma 2.6]). Since the inclusion $C_{u}^{*}(X) \hookrightarrow C_{u q}^{*}(X)$ is nuclear, there exist unital completely positive maps $\phi: C_{u}^{*}(X) \rightarrow M_{n}(\mathbb{C})$ and $\psi: M_{n}(\mathbb{C}) \rightarrow C_{u q}^{*}(X)$ such that $\|(\psi \circ \phi)(v)-v\|<\varepsilon$ for all $v \in \mathcal{F}$ (see also [5, Proposition 2.2.6]).

Denoting by $\left\{e_{i j}\right\}_{1 \leq i, j \leq n}$ the matrix units of $M_{n}(\mathbb{C})$, the matrix $\left[\psi\left(e_{i j}\right)\right]_{i, j=1}^{n}$ is positive in $M_{n}\left(C_{u q}^{*}(X)\right)\left[\underline{5}\right.$, Proposition 1.5.12]. Let $\left[b_{i j}\right]=\left[\psi\left(e_{i j}\right)\right]^{1 / 2} \in M_{n}\left(C_{u q}^{*}(X)\right)$ and denote by $\left\{\xi_{i}\right\}_{1 \leq i \leq n}$ the standard basis for $\mathbb{C}^{n}$. We define

$$
\xi_{\psi}=\sum_{j, k=1}^{n} \xi_{j} \otimes \xi_{k} \otimes b_{k j} \in \mathbb{C}^{n} \otimes \mathbb{C}^{n} \otimes C_{u q}^{*}(X) .
$$

Note that $\mathbb{C}^{n} \otimes \mathbb{C}^{n} \otimes C_{u q}^{*}(X)$ is a Hilbert $C_{u q}^{*}(X)$-module equipped with an inner product $\langle\cdot, \cdot\rangle$ defined by

$$
\left\langle\xi \otimes \eta \otimes T, \xi^{\prime} \otimes \eta^{\prime} \otimes T^{\prime}\right\rangle=\left\langle\xi, \xi^{\prime}\right\rangle_{\mathbb{C}^{n}} \cdot\left\langle\eta, \eta^{\prime}\right\rangle_{\mathbb{C}^{n}} \cdot T^{*} T^{\prime}
$$

for elementary tensor elements in $\mathbb{C}^{n} \otimes \mathbb{C}^{n} \otimes C_{u q}^{*}(X)$, where $\langle\cdot, \cdot\rangle_{\mathbb{C}^{n}}$ is the standard inner product on $\mathbb{C}^{n}$ which is linear in the second variable, then we extend it linearly to general elements in $\mathbb{C}^{n} \otimes \mathbb{C}^{n} \otimes C_{u q}^{*}(X)$. Note that $M_{n}(\mathbb{C}) \otimes M_{n}(\mathbb{C})$ acts on (the 
first two tensor factors of) $\mathbb{C}^{n} \otimes \mathbb{C}^{n} \otimes C_{u q}^{*}(X)$. With this action, it is straightforward to check that for any $A \in M_{n}(\mathbb{C})$ we have

$$
\psi(A)=\left\langle\xi_{\psi},\left(A \otimes 1_{n}\right) \xi_{\psi}\right\rangle_{C_{u q}^{*}(X)},
$$

which implies that $\left\|\xi_{\psi}\right\|_{C_{u q}^{*}(X)}=1$ (choosing $A=1$ ). Denoting by $\left\{\eta_{l}\right\}_{1 \leq l \leq n^{2}}$ the standard basis for $\mathbb{C}^{n^{2}} \cong \mathbb{C}^{n} \otimes \mathbb{C}^{n}$, we write

$$
\xi_{\psi}=\sum_{l=1}^{n^{2}} \eta_{l} \otimes a_{l} \in \mathbb{C}^{n^{2}} \otimes C_{u q}^{*}(X) .
$$

Now we define a map $\zeta: X \rightarrow \ell^{2}(X)$ by

$$
\zeta_{x}(z)=\left\|\sum_{l=1}^{n^{2}} \eta_{l}\left\langle\delta_{z}, a_{l} \delta_{x}\right\rangle_{\ell^{2}(X)}\right\|_{\mathbb{C}^{n^{2}}}
$$

We proceed analogously to the argument in the proof of [5, Theorem 5.5.7] to show that $\zeta$ satisfies the conditions (a) and (b) from Lemma 5.3(2). For the convenience of readers, we present the details here as well. For any $x \in X$, we have:

$$
\begin{aligned}
\left\|\zeta_{x}\right\|^{2} & =\sum_{z}\left\|\sum_{l} \eta_{l}\left\langle\delta_{z}, a_{l} \delta_{x}\right\rangle_{\ell^{2}(X)}\right\|_{\mathbb{C}^{n^{2}}}^{2} \\
& =\sum_{l, k} \sum_{z}\left\langle\eta_{l}, \eta_{k}\right\rangle_{\mathbb{C}^{n^{2}}} \overline{\left\langle\delta_{z}, a_{l} \delta_{x}\right\rangle_{\ell^{2}(X)}}\left\langle\delta_{z}, a_{k} \delta_{x}\right\rangle_{\ell^{2}(X)} \\
& =\sum_{l, k}\left\langle\eta_{l}, \eta_{k}\right\rangle_{\mathbb{C}^{n^{2}}}\left\langle a_{l} \delta_{x}, a_{k} \delta_{x}\right\rangle_{\ell^{2}(X)} \\
& =\left\langle\delta_{x},\left\langle\xi_{\psi}, \xi_{\psi}\right\rangle_{\mathbb{C}^{n^{2} \otimes C_{u q}^{*}(X)}} \delta_{x}\right\rangle_{\ell^{2}(X)}=1 .
\end{aligned}
$$

On the other hand, let $d(x, y) \leq R$ and choose $v \in \mathcal{F}$ such that $v \delta_{x}=\delta_{y}$. Using that $\phi(v)$ is contractive and (5.1) with $A=\phi(v)$, we have that

$$
\begin{aligned}
\left\langle\zeta_{y}, \zeta_{x}\right\rangle & =\sum_{z}\left\|\sum_{l} \eta_{l}\left\langle\delta_{z}, a_{l} \delta_{y}\right\rangle\right\|_{\mathbb{C}^{n^{2}}} \cdot\left\|\sum_{k} \eta_{k}\left\langle\delta_{z}, a_{k} \delta_{x}\right\rangle\right\|_{\mathbb{C}^{n^{2}}} \\
& \geq \sum_{z}\left\|\sum_{l} \eta_{l}\left\langle\delta_{z}, a_{l} \delta_{y}\right\rangle\right\|_{\mathbb{C}^{n^{2}}} \cdot\left\|(\phi(v) \otimes 1) \sum_{k} \eta_{k}\left\langle\delta_{z}, a_{k} \delta_{x}\right\rangle\right\|_{\mathbb{C}^{n^{2}}} \\
& \geq\left|\sum_{l, k} \sum_{z}\left\langle\eta_{l},(\phi(v) \otimes 1) \eta_{k}\right\rangle_{\mathbb{C}^{n^{2}}} \cdot \frac{\left\langle\delta_{z}, a_{l} \delta_{y}\right\rangle_{\ell^{2}(X)}}{} \cdot\left\langle\delta_{z}, a_{k} \delta_{x}\right\rangle_{\ell^{2}(X)}\right| \\
& =\left|\left\langle\delta_{y},\left\langle\xi_{\psi},(\phi(v) \otimes 1) \xi_{\psi}\right\rangle_{\mathbb{C}^{n} \otimes \mathbb{C}^{n} \otimes C_{u q}^{*}(X)} \delta_{x}\right\rangle_{\ell^{2}(X)}\right|=\left|\left\langle\delta_{y},(\psi \circ \phi)(v) \delta_{x}\right\rangle_{\ell^{2}(X)}\right| \\
& \geq\left|\left\langle\delta_{y}, v \delta_{x}\right\rangle_{\ell^{2}(X)}\right|-\varepsilon=1-\varepsilon .
\end{aligned}
$$

This implies that $\left\|\zeta_{x}-\zeta_{y}\right\|$ is sufficiently small.

We also claim:

$$
\lim _{S \rightarrow \infty} \sup _{x \in X} \sum_{y \notin B(x, S)}\left|\zeta_{x}(y)\right|^{2}=0 .
$$

In fact, by definition we have

$$
\left|\zeta_{x}(y)\right|^{2}=\sum_{l=1}^{n^{2}}\left|\left\langle\delta_{y}, a_{l} \delta_{x}\right\rangle\right|^{2} .
$$


Note that for all $1 \leq l \leq n^{2}, a_{l}$ is quasi-local by assumption. Hence given any $\varepsilon^{\prime}>0$, there exists an $S>0$ such that for all $x \in X$, we have

$$
\left\|\chi_{B(x, S)^{c}} a_{l} \chi_{\{x\}}\right\|^{2}=\left.\sum_{y \notin B(x, S)}\left\langle\delta_{y}, a_{l} \delta_{x}\right\rangle\right|^{2}<\varepsilon^{\prime} / n^{2},
$$

which implies that $\sup _{x \in X} \sum_{y \notin B(x, S)}\left|\zeta_{x}(y)\right|^{2}<\varepsilon^{\prime}$.

In conclusion, we have shown that the function $\zeta$ constructed above satisfies also condition (c) from Lemma 5.3(2). Hence due to Lemma 5.3 we are done.

Remark 5.6. Let $X$ be a metric space with bounded geometry such that $X$ admits a coarse embedding into a countable discrete group. Then additionally, conditions (1) $\sim(5)$ in the above theorem are also equivalent to: $(6) C_{u q}^{*}(X)$ is exact. Indeed, this follows directly from [4, Corollary 30] and the facts that nuclearity implies exactness, and exactness is preserved under taking $C^{*}$-subalgebras (we refer readers to [5] for the relevant concepts).

Remark 5.7. Let $G$ be a finitely generated residually finite group and $X$ be any of its box spaces. Then additionally, conditions $(1) \sim(5)$ in the above theorem are also equivalent to: $(6) C_{u q}^{*}(X)$ is exact; $(7) C_{u q}^{*}(X)$ is locally reflexive. Indeed, since nuclearity implies exactness and exactness implies locally reflexivity, it remains to prove condition (7) implies $X$ having Property A. Suppose not, then $X$ is a weak expander by [27, Lemma 2.6]. In particular, the uniform Roe algebra $C_{u}^{*}(X)$ is not locally reflexive (see [27, Theorem 1.1]). Since locally reflexivity is preserved under taking $C^{*}$-subalgebras, we conclude that $C_{u q}^{*}(X)$ is not locally reflexive as well (see [5, Chapter 9] for more details).

Finally, we record here that if the box space $X$ is a sequence of asymptotic expanders then $X$ must be a weak expander (see Theorem 4.4, [3, Theorem 4.5] and [27, Lemma 2.6]).

Remark 5.8. Very recently, Sako proved in [28] a remarkable result that for a metric space $X$ with bounded geometry, $X$ has Property A if and only if $C_{u}^{*}(X)$ is exact if and only if $C_{u}^{*}(X)$ is locally reflexive. Therefore combining Theorem 5.5 with Sako's result, we conclude that for a general metric space with bounded geometry, conditions $(1) \sim(7)$ above are all equivalent.

\section{Cartan subalgebras in Uniform QUASI-LOCAL Algebras}

The main result of this section is Proposition 6.1, which provides another take on the question when $C_{u}^{*}(X)=C_{u q}^{*}(X)$ in the context of Cartan subalgebras of these algebras.

Recall that a pair of $C^{*}$-algebras $\mathcal{B} \subseteq \mathcal{A}$ is a Cartan pair (or $\mathcal{B}$ is a Cartan subalgebra of $\mathcal{A}$ ) [22] if $\mathcal{B}$ is a maximal abelian self-adjoint subalgebra containing an approximate unit of $\mathcal{A}$ such that the normaliser of $\mathcal{B}$ inside $\mathcal{A}$ generates $\mathcal{A}$ as a $C^{*}$-algebra, and there exists a faithful conditional expectation $E: \mathcal{A} \rightarrow \mathcal{B}$. Here the normaliser of $\mathcal{B}$ in $\mathcal{A}$ is defined as $\left\{a \in \mathcal{A}: a \mathcal{B} a^{*} \cup a^{*} \mathcal{B} a \subseteq \mathcal{B}\right\}$. It is clear that $\ell^{\infty}(X) \subseteq C_{u q}^{*}(X)$ is a Cartan pair if and only if the normaliser of $\ell^{\infty}(X)$ in $C_{u q}^{*}(X)$ generates $C_{u q}^{*}(X)$. Moreover, $\ell^{\infty}(X) \subseteq C_{u}^{*}(X)$ is always a Cartan pair.

Proposition 6.1. Let $X$ be a metric space with bounded geometry. Then the following are equivalent: 
(1) $C_{u}^{*}(X)=C_{u q}^{*}(X)$;

(2) $\ell^{\infty}(X) \subseteq C_{u q}^{*}(X)$ is a Cartan pair;

(3) the normaliser of $\ell^{\infty}(X)$ in $C_{u q}^{*}(X)$ generates $C_{u q}^{*}(X)$.

To prove it, we need the following lemma analysing the normalisers of $\ell^{\infty}(X)$ in $C_{u}^{*}(X)$ and $C_{u q}^{*}(X)$ :

Lemma 6.2. Let $X$ be a metric space with bounded geometry. Then the normalisers of $\ell^{\infty}(X)$ in $C_{u}^{*}(X)$ and in $C_{u q}^{*}(X)$ are the same. More precisely, the following are equivalent for $T \in \mathfrak{B}\left(\ell^{2}(X)\right)$ :

(1) $T$ belongs to the normaliser of $\ell^{\infty}(X)$ in $C_{u}^{*}(X)$;

(2) $T$ belongs to the normaliser of $\ell^{\infty}(X)$ in $C_{u q}^{*}(X)$;

(3) $T=f V^{\theta}$ for some $f \in \ell^{\infty}(X)$ and some bijection $\theta: D \rightarrow R$ where $D, R \subseteq$ $X$ satisfying: for any $\varepsilon>0$, there exists some $K>0$ such that for any $x \in D$ with $d(x, \theta(x))>K$ we have $|f(x)|<\varepsilon$.

Proof. It is straightforward to check that for $T=f V^{\theta}$ satisfying condition (3), we have $T \in C_{u}^{*}(X) \subseteq C_{u q}^{*}(X)$, and it normalises $\ell^{\infty}(X)$. This implies " $(3) \Rightarrow(1)$ " and "(3) $\Rightarrow(2) "$.

For the other directions, note that any element in the normaliser of $\ell^{\infty}(X)$ in $\mathfrak{B}\left(\ell^{2}(X)\right)$ has the form of $T=f V^{\theta}$ for some $f \in \ell^{\infty}(X)$ and bijection $\theta: D \rightarrow R$ for $D, R \subseteq X$. Since $\|T\|=\sup _{x \in X}|f(x)|$, one can check directly that $T=f V^{\theta}$ is quasi-local if and only if for any $\varepsilon>0$, there exists some $K>0$ such that if $x \in D$ with $d(x, \theta(x))>K$ then $|f(x)|<\varepsilon$. The same condition also implies that $f V^{\theta}$ can be approximated in norm by operators with finite propagation using the fact that $\|T\|=\sup _{x \in X}|f(x)|$ again.

Proof of Proposition 6.1. Since $\ell^{\infty}(X) \subseteq C_{u}^{*}(X)$ is a Cartan pair, we have "(1) $\Rightarrow$ $(2) \Rightarrow(3)$ ". Now condition (3) says that $C_{u q}^{*}(X)$ is generated by the normaliser of $\ell^{\infty}(X)$ in $C_{u q}^{*}(X)$, which coincides with the normaliser of $\ell^{\infty}(X)$ in $C_{u}^{*}(X)$ by Lemma 6.2. Since $\ell^{\infty}(X) \subseteq C_{u}^{*}(X)$ is a Cartan pair, condition (1) holds.

\section{Open questions}

According to Proposition 2.4 and Proposition 6.1, we may ask the following natural question:

Question 7.1. Let $X$ be a metric space with bounded geometry. Suppose that $\ell^{\infty}(X)$ is a Cartan subalgebra of the uniform quasi-local algebra $C_{u q}^{*}(X)$. Does $X$ have Property A?

Let $\left\{Y_{n}\right\}_{n \in \mathbb{N}}$ be the sequence of asymptotic expander graphs in Example 3.7 (see also Corollary 3.13). Since the averaging projection $P_{Y}$ sits inside the uniform Roe algebra $C_{u}^{*}(Y)$ for the coarse disjoint union $Y=\bigsqcup_{n \in \mathbb{N}} Y_{n}$, it follows that $Y$ does not satisfy the coarse Baum-Connes conjecture provided that $\left\{Y_{n}\right\}_{n \in \mathbb{N}}$ has large girth (see [14, [15] and [39, Theorem 6.1]). Does this conclusion hold generally?

Question 7.2. If $\left\{Y_{n}\right\}_{n \in \mathbb{N}}$ is any sequence of asymptotic expander graphs with large girth and let $Y$ be its coarse disjoint union, does the coarse Baum-Connes conjecture for $Y$ fail? 
We now turn to the relation between asymptotic expanders and coarse embeddability. It is well known that a sequence of expander graphs can not be coarsely embedded into any Hilbert space (see e.g. [20, Theorem 5.6.5]).

Question 7.3. Let $X$ be a coarse disjoint union of asymptotic expanders $\left\{X_{n}\right\}_{n \in \mathbb{N}}$ with bounded geometry. Can $X$ be coarsely embedded into some Hilbert space?

This question has a negative answer with an extra hypothesis:

Proposition 7.4. Let $X$ be a coarse disjoint union of asymptotic expanders with bounded geometry. If $C_{u}^{*}(X)=C_{u q}^{*}(X)$, then $X$ can not be coarsely embedded into any Hilbert space.

Proof. It follows from the hypothesis and Theorem 3.11 that the averaging projection $P_{X}$ belongs to the uniform Roe algebra $C_{u}^{*}(X)$. On the other hand, if $X$ can be coarsely embedded into a Hilbert space, then the Roe algebra $C^{*}(X)$ (and hence also the uniform Roe algebra $\left.C_{u}^{*}(X)\right)$ does not possess any non-compact ghost projection by [11, Proposition 35] and [45, Theorem 1.1]. Since $P_{X}$ is always a non-compact ghost projection, we complete the proof.

If Question 7.3 has an affirmative answer (i.e., there exists a sequence of asymptotic expanders which can be coarsely embedded into some Hilbert space), then from Proposition 7.4 we would provide an example of a space $X$ such that the uniform Roe algebra $C_{u}^{*}(X)$ is properly contained in the uniform quasi-local algebra $C_{u q}^{*}(X)$, which answers Question 6.7 in [34].

\section{REFERENCES}

[1] Pere Ara, Kang Li, Fernando Lledó, and Jianchao Wu. Amenability and uniform Roe algebras. Journal of Mathematical Analysis and Applications, 459(2):686-716, 2018.

[2] Christian Bönicke and Kang Li. Ideal structure and pure infiniteness of ample groupoid $C^{*}$ algebras. Ergodic Theory Dynam. Systems, 40(1):34-63, 2020.

[3] Jacek Brodzki, Graham A. Niblo, Ján Špakula, Rufus Willett, and Nick Wright. Uniform local amenability. J. Noncommut. Geom., 7(2):583-603, 2013.

[4] Jacek Brodzki, Graham A. Niblo, and Nick J. Wright. Property A, partial translation structures, and uniform embeddings in groups. J. Lond. Math. Soc. (2), 76(2):479-497, 2007.

[5] Nathanial P. Brown and Narutaka Ozawa. $\mathrm{C}^{*}$-algebras and finite-dimensional approximations, volume 88 of Graduate Studies in Mathematics. American Mathematical Society, Providence, RI, 2008.

[6] Xiaoman Chen, Romain Tessera, Xianjin Wang, and Guoliang Yu. Metric sparsification and operator norm localization. Adv. Math., 218(5):1496-1511, 2008.

[7] Xiaoman Chen and Qin Wang. Ideal structure of uniform Roe algebras of coarse spaces. Journal of Functional Analysis, 216(1):191 - 211, 2004.

[8] Yeong Chyuan Chung and Kang Li. Structure and K-theory of $\ell^{p}$-uniform Roe algebras. arXiv:1904.07050, to appear in Journal of Noncommutative Geometry, 2019.

[9] Giuliana Davidoff, Peter Sarnak, and Alain Valette. Elementary number theory, group theory, and Ramanujan graphs, volume 55 of London Mathematical Society Student Texts. Cambridge University Press, Cambridge, 2003.

[10] Alexander Engel. Rough index theory on spaces of polynomial growth and contractibility. $J$. Noncommut. Geom., 13(2):617-666, 2019.

[11] Martin Finn-Sell. Fibred coarse embeddings, a-T-menability and the coarse analogue of the Novikov conjecture. J. Funct. Anal., 267(10):3758-3782, 2014.

[12] Rostislav Grigorchuk. Some topics in the dynamics of group actions on rooted trees. Proceedings of the Steklov Institute of Mathematics, 273:64-175, 2011.

[13] Erik Guentner and Jerome Kaminker. Exactness and the Novikov conjecture. Topology, 41(2):411-418, 2002. 
[14] Nigel Higson. Counterexamples to the coarse Baum-Connes conjecture. Available on the author's website, 1999.

[15] Nigel Higson, Vincent Lafforgue, and Georges Skandalis. Counterexamples to the BaumConnes conjecture. Geom. Funct. Anal., 12(2):330-354, 2002.

[16] Ana Khukhro and Alain Valette. Expanders and box spaces. Adv. Math., 314:806-834, 2017.

[17] Kang Li and Hung-Chang Liao. Classification of uniform Roe algebras of locally finite groups. J. Operator Theory, 80(1):25-46, 2018.

[18] Kang Li and Rufus Willett. Low-dimensional properties of uniform Roe algebras. Journal of the London Mathematical Society, 97(1):98-124, 2018.

[19] G. A. Margulis. Explicit constructions of expanders. Problemy Peredači Informacii, 9(4):7180, 1973.

[20] Piotr W. Nowak and Guoliang Yu. Large scale geometry. EMS Textbooks in Mathematics. European Mathematical Society (EMS), Zürich, 2012.

[21] Narutaka Ozawa. Amenable actions and exactness for discrete groups. C. R. Acad. Sci. Paris Sér. I Math., 330(8):691-695, 2000.

[22] Jean Renault. Cartan subalgebras in $C^{*}$-algebras. Irish Math. Soc. Bull., (61):29-63, 2008.

[23] John Roe. An index theorem on open manifolds. I, II. J. Differential Geom., 27(1):87-113, 115-136, 1988.

[24] John Roe. Index theory, coarse geometry, and topology of manifolds, volume 90 of CBMS Regional Conference Series in Mathematics. Published for the Conference Board of the Mathematical Sciences, Washington, DC; by the American Mathematical Society, Providence, RI, 1996.

[25] John Roe. Lectures on coarse geometry, volume 31 of University Lecture Series. American Mathematical Society, Providence, RI, 2003.

[26] John Roe and Rufus Willett. Ghostbusting and property A. J. Funct. Anal., 266(3):1674-1684, 2014.

[27] Hiroki Sako. A generalization of expander graphs and local reflexivity of uniform Roe algebras. J. Funct. Anal., 265(7):1367-1391, 2013.

[28] Hiroki Sako. Finite dimensional approximation properties for uniform Roe algebras. arXiv preprint arXiv:1212.5900v3, 2019.

[29] Damian Sawicki. Super-expanders and warped cones. to appear in Annales de l'Institut Fourier, 2017.

[30] Georges Skandalis, Jean-Louis Tu, and Guoliang Yu. The coarse Baum-Connes conjecture and groupoids. Topology, 41(4):807-834, 2002.

[31] Federico Vigolo. Geometry of actions, expanders and warped cones. PhD thesis, University of Oxford, 2018.

[32] Ján Špakula. Uniform K-homology theory. J. Funct. Anal., 257(1):88-121, 2009.

[33] Ján Špakula and Aaron Tikuisis. Relative commutant pictures of Roe algebras. Comm. Math. Phys., 365(3):1019-1048, 2019.

[34] Ján Špakula and Jiawen Zhang. Quasi-locality and Property A. Journal of Functional Analysis, 278(1):108299, 2020.

[35] Qin Wang. Remarks on ghost projections and ideals in the Roe algebras of expander sequences. Arch. Math. (Basel), 89(5):459-465, 2007.

[36] ShuYun Wei. On the quasidiagonality of Roe algebras. Sci. China Math., 54(5):1011-1018, 2011.

[37] Stuart White and Rufus Willett. Cartan subalgebras in uniform Roe algebras. arXiv:1808.04410, to appear in Groups, Geometry, and Dynamics, 2018.

[38] Rufus Willett. Some notes on property A. In Limits of graphs in group theory and computer science, pages 191-281. EPFL Press, Lausanne, 2009.

[39] Rufus Willett and Guoliang Yu. Higher index theory for certain expanders and Gromov monster groups, I. Adv. Math., 229(3):1380-1416, 2012.

[40] Wilhelm Winter and Joachim Zacharias. The nuclear dimension of $C^{*}$-algebras. Adv. Math., 224(2):461-498, 2010.

[41] Guoliang Yu. Coarse Baum-Connes conjecture. K-Theory, 9(3):199-221, 1995.

[42] Guoliang Yu. Localization algebras and the coarse Baum-Connes conjecture. K-Theory, 11(4):307-318, 1997. 
[43] Guoliang Yu. Zero-in-the-spectrum conjecture, positive scalar curvature and asymptotic dimension. Invent. Math., 127(1):99-126, 1997.

[44] Guoliang Yu. The Novikov conjecture for groups with finite asymptotic dimension. Ann. of Math. (2), 147(2):325-355, 1998.

[45] Guoliang Yu. The coarse Baum-Connes conjecture for spaces which admit a uniform embedding into Hilbert space. Invent. Math., 139(1):201-240, 2000.

(K. Li) Institute of Mathematics of the Polish Academy of Sciences, Śniadeckich 8, 00-656 WarsaW, POLAND.

E-mail address: kli@impan.pl

(P. Nowak) Institute of Mathematics of the Polish Academy of Sciences, Śniadeckich 8, 00-656 Warsaw, Poland.

E-mail address: pnowak@impan.pl

(J. Špakula) School of Mathematics, University of Southampton, Highfield, SO17 1BJ, United Kingdom.

E-mail address: jan.spakula@soton.ac.uk

(J. Zhang) School of Mathematics, University of Southampton, Highfield, SO17 1BJ, United Kingdom.

E-mail address: jiawen.zhang@soton.ac.uk 